\title{
Does the Way in Which We Perceive the World Makes Us Susceptible to Anxiety?
}

\author{
Billy Jansson
}

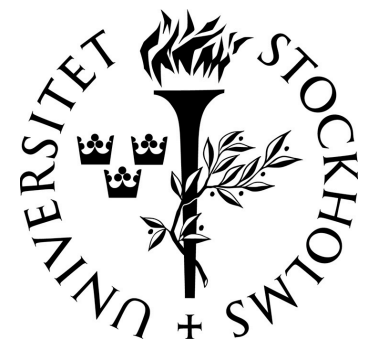

Department of Psychology

Stockholm University

Sweden 
(C) 2005 Billy Jansson

ISBN 91-7155-181-6

Akademitryck AB, Valdemarsvik, 2005 
Doctoral Dissertation, 2006

Department of Psychology

Stockholm University

S-106 91 Stockholm

\begin{abstract}
One major focus of anxiety research in recent years has been the identification of cognitive factors that promote increased vulnerability to anxiety. Cognitive formulations propose that anxiety is characterised by an increased tendency to attend to negatively valenced emotional information, and that this bias may play a causal role in the development and maintenance of clinical anxiety. Evidence suggests that this anxiety-linked processing bias occurs even in conditions in which the stimuli are masked in order to prevent awareness of the content (i.e., preattentive bias). The present thesis focused principally on the preferential processing of threat-related information that occurs outside awareness. Two different outcome measures were used to index preferential preattentive processing of threat-related information in non-clinical populations: The emotional Stroop task was used to index selective attention to masked presentation of threatening word stimuli, whereas skin conductance responses were used to index selective autonomic responses to masked presentation of threatening pictorial stimuli. The empirical studies in the present thesis showed that elevated levels of trait anxiety promote preferential preattentive processing of negatively valenced information, whereas elevated levels of social desirability (interpreted as defensiveness) generally prevent preferential preattentive processing of negatively valenced information, especially among those at higher levels of trait anxiety, irrespective of outcome measure used. Moreover, previous research has demonstrated that a person's most common emotional reaction when encountering a stressful event is causally influenced by that person's habitual tendency to selectively encode the most threatening aspects of all available information. Thus, preattentive bias (as measured with the emotional Stroop task) was used to predict the emotional responses (as seen on self-reported emotional distress and autonomic reactivity) following exposure to a laboratory stressor. This study showed that preattentive bias to negative information had significant effects on both self-reported and physiological measures in response to a laboratory stressor, but in diametrically opposite directions. Specifically, whereas preattentive bias was positively associated with self-reported negative emotional response, it was negatively associated with a physiological indicator of emotional response. The results were discussed in terms of an inability to automatically inhibit the processing of threatening cues, which seems to be a vulnerability marker for anxiety. Whether this bias is ultimately sufficient for the development of clinical anxiety remains to be examined in future research. Additionally, more information is needed before it can be established that the emotional Stroop task can be viewed as a reliable diagnostic tool for determining an individual's anxiety status.
\end{abstract}

Key words. Preattentive processing, selective attention, trait anxiety, defensiveness, emotional responses, emotional vulnerability. 
The present thesis is based on the following studies:

I. Jansson, B., Lundh, L. G., \& Oldenburg, C. (2005). Is defensiveness associated with cognitive bias away from emotional information? Personality and Individual Differences, 39, 1373-1382.

II. Najström, M., \& Jansson, B. (in press). Unconscious responses to threatening pictures: The interactive effect of trait anxiety and social desirability on skin conductance responses. Cognitive Behaviour Therapy.

III. Jansson, B., \& Lundh, L. G. (submitted for publication). The interactive role of worried mood and trait anxiety in the selective processing of subliminally presented threat words

IV. Jansson, B., \& Najström, M. (submitted for publication). Informationprocessing and emotional responses to stress: An investigation using self-report and physiological measures of stress.

Study I was printed with permission from Taylor \& Francis.

Study II was printed with permission from Elsevier Science. 
To my family and friends 


\section{Acknowledgement}

My last remaining task in writing this thesis is to acknowledge all the people who made it possible. This is an impossible task - I do know who made a direct contribution, but I do not know everyone who made an indirect contribution. I am going to do it anyway, and if your name is not listed, rest assured that my appreciation for you is not less than for those listed below.

First and foremost I would like to thank my former supervisor and co-author LarsGunnar Lundh, who has shown a great and consistent interest in my project all along. His many constructive comments have proved valuable to my work. I am also thankful to my supervisor, Lars-Göran Öst, not only for his thorough review and valuable comments on an earlier draft on this manuscript, but also for being helpful in different ways. The contribution of Mats Najström, who has been my co-worker on some projects, should, of course, be acknowledged. Apart from our collaborative work, I have also appreciated our scientific discussions, as well as our even more frequent "not-so-scientific" discussions. I would also like to thank Maria Larsson and Gerhard Andersson for their comments on an earlier draft of this manuscript.

In my daily work I have been blessed with friendly people, both in the past and present. I want to express my gratitude to my colleagues, office neighbours and friends at the department for creating a pleasant and stimulating work environment, as well as for providing valuable and enriching discussions. I particularly want to express my gratitude to my long-time companions during these years, Göran Söderlund and Jonas Ramnerö. I also want to express my appreciation to Lisa Breitholtz, Hi-Young Kim, Kimmo Sorjonen, Jan Dalkvist, Ann-Charlotte Smedler, Bertil Törestad, Bertil Haarala, Jenny Wikström and Maggan. Of course, thanks to all of you who participated in the studies. Finally, it is customary to mention the contributions of family and friends, and I will make no exception to this custom thus, thank you all! 


\section{Contents}

Introduction ................................................................ 1

Selective attention to threatening information.................................................... 1

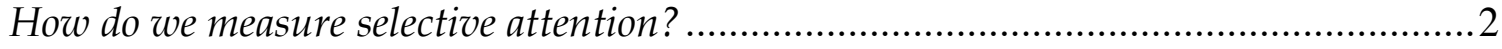

Selective attention: Conscious or an unconscious phenomenon? ............................5

Mechanisms underlying selective attention towards threat................................ 8

At times, people selectively attend away from threatening information ................ 10

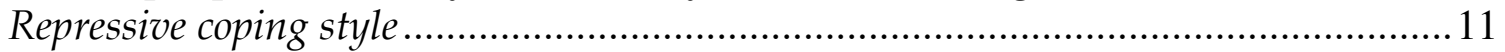

What is the functional significance of selective attention away from negative

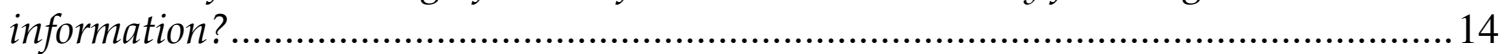

Why study selective attention in non-clinical populations, and why use the

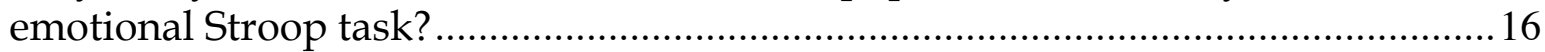

Selective attention to unconsciously perceived information as a factor in the development

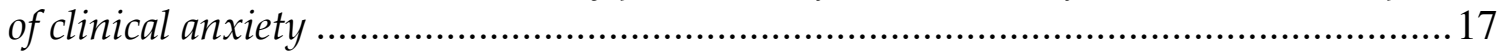

The empirical studies ............................................. 18

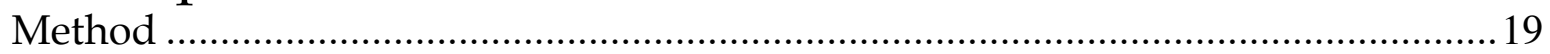

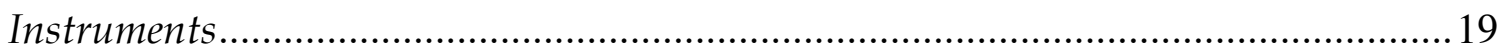

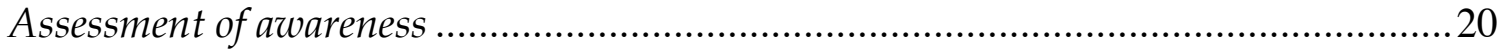

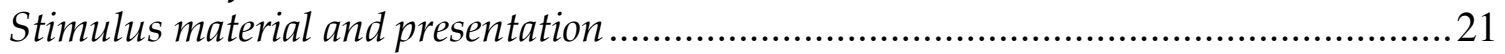

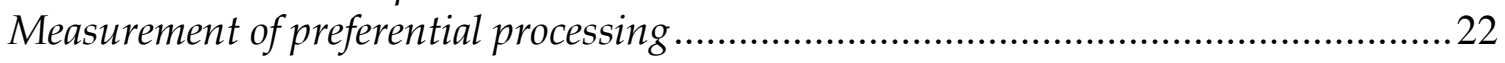

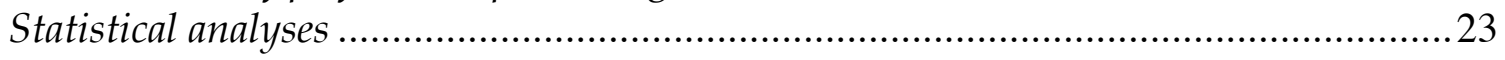

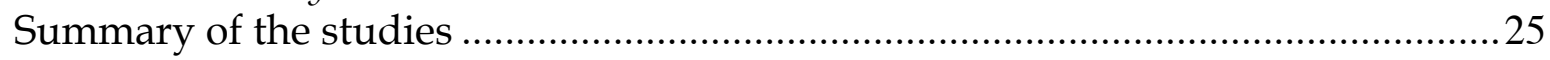

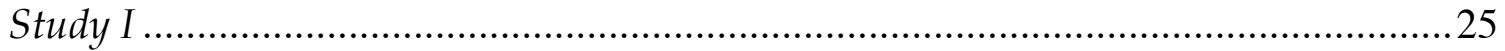

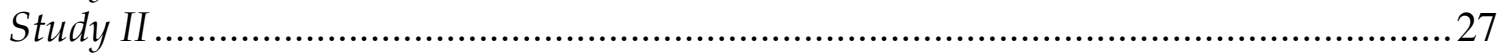

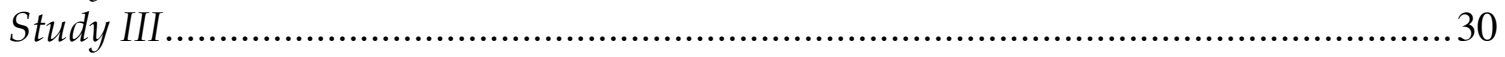

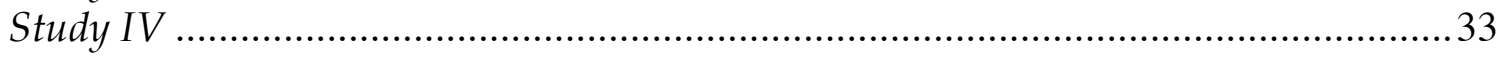

General discussion ...................................................37

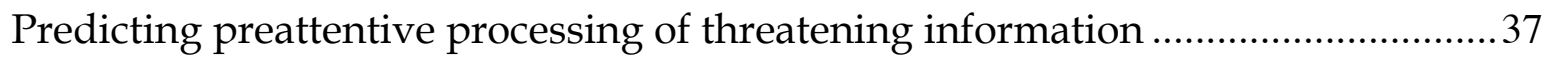

Predicting emotional responses to a stressful event.............................................39

Is the preattentive bias for threat a vulnerability factor in the development of anxiety

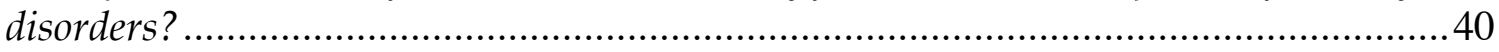

The importance of trait anxiety and social desirability in preferential preattentive

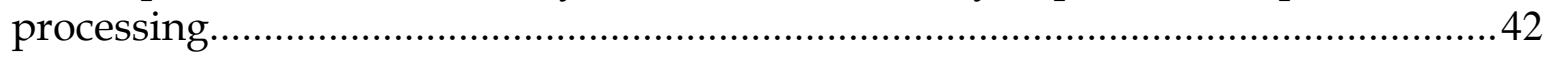

Temporal stability of Stroop interference indices .......................................... 44

Preferential preattentive processing: An extended discussion.................................45

Subliminal vs. supraliminal: How much attention should we pay to effects seen in

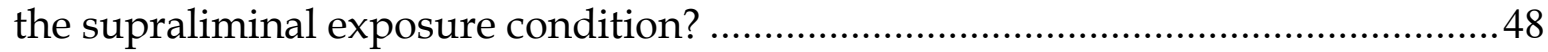

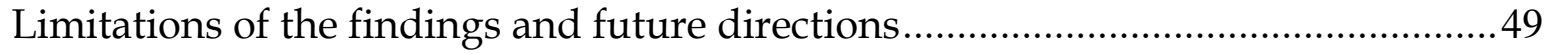

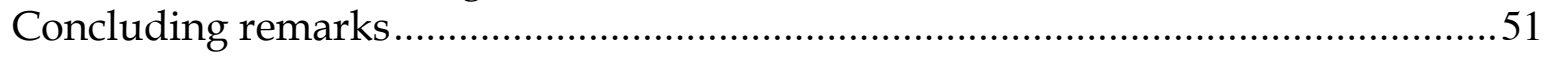

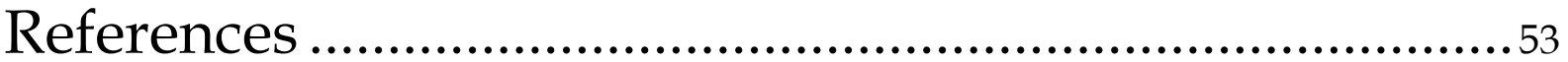





\section{Introduction}

The world that we live in is inherently variable. Every day we are faced with an overpowering quantity of information, but we simply cannot attend to all of it. To navigate through the constant and diverse stimuli humans confront everyday in their environment, attentional processes are vital for selecting and evaluating significant information to be processed further, as we usually assign selective attention to one type of information at the expense of another based on our current motivational state (Lang, Bradley, \& Cuthbert, 1998). In most cases, we do so without awareness of the fact that a particular piece of information has been actively processed. In addition, some specific information may also have different emotional consequences for different persons. The study of a phenomenon such as selective attention has been largely confined to the laboratory. We cannot blend the control of the laboratory with the richness of the everyday world in order to achieve a better understanding of the phenomenon as such. When moving findings from the laboratory to the everyday world, however, we are perhaps likely to have insight into how these findings correspond to everyday situations. This thesis attempts to deal with issues that have been answered insufficiently in previous literature concerned with selective attention.

\section{Selective attention to threatening information}

In the past two decades, the cognitive perspective in research on emotions has become the chief approach in psychology (e.g., Lazarus, 1999). According to recent cognitive theories of anxiety disorders, anxious individuals process threat-related information in a biased way (e.g., Beck \& Clark, 1997; Rapee \& Heimberg, 1997), and to date there is also much evidence demonstrating that selective attention is in fact associated with anxiety. To be precise, empirical work has provided strong evidence confirming that clinically anxious individuals, as well as non-clinical individuals with elevated levels of anxiety, selectively attend to threat-related stimuli at the expense of neutral stimuli (e.g., Williams, Watts, MacLeod \& Mathews, 1997; Mogg \& Bradley, 1998, for reviews). In general, with respect to clinically anxious individuals, these effects appear to be strongest when the stimuli match their particular fear (Williams, Mathews, \& MacLeod, 1996). With regard to the stimulus content for nonclinically highly anxious individuals, the effects are more non-specific in their nature, and may therefore be sensitive to people who are reactive to a broader spectrum of negative stimuli. In fact, individuals who score high on measures of trait anxiety have been suggested as likely candidates among those who are disposed to respond with fear and anxiety to a wide range of stressors (e.g., McNally, 1989).

One main question has, of course, been whether selective attention to threatening information is best described as an effect of stable personality characteristics such as trait anxiety, or whether temporary anxious states play the key role in determining 
the outcome. Even though biased processing is typically seen in individuals with elevated levels of enduring anxiety, state variables have also been found to exert influence on the response patterns. For example, Chen, Lewin and Craske (1996) found a significant effect of selective attention to spider-related threat cues among spider fearful subjects, and this effect was further enhanced when these individuals were stressed (induced by exposure to a spider). Furthermore, Egloff and Hock (2001) reported that elevated levels of stress among high trait-anxious individuals were necessary in order to observe significant effects of selective attention to threatening material. The net sum is, however, that the effects are dependent on enduring personality characteristics rather than something that occurs to everyone when in a temporary state of anxious mood. Selective attentional processing has received additional attention because recent cognitive formulations (e.g., Eysenck, 1997; Williams et al., 1997) have placed much emphasis on selective attention to threatening material as a key cognitive factor underlying the development and maintenance of anxiety disorders. Thus, if people were to display a tendency to selectively attend to negative information in a situation in which positive information was equally available, this could then directly influence their emotional vulnerability by increasing the likelihood that they would experience a negative emotional reaction to that particular situation. This in turn, may function to further strengthen the selective processing in favour of negative information in future situations. As a consequence, any tendency to selectively process negative information may directly have an impact upon the likelihood of an impending emotional disorder. To date, an equal amount of interest in investigating the selectivity of attentional processes has been directed at individuals who are not clinically anxious. Though it has been suggested that various trait factors, such as negative affectivity (e.g., Clark, Watson, \& Mineka, 1994) and anxiety sensitivity (McNally, 1990) may play a key role in the development of clinical anxiety, selective attention and trait factors are by no means independent of each other. We need to fully understand how attentional processes operate in general, and more specifically how the selective processing of emotional information operates in non-clinical populations in order to tease apart those factors that may be responsible for the maintenance and development of anxiety disorders.

\section{How do we measure selective attention?}

How might one then measure selective attention? Studies have typically been of two types: one based on measurement of spatial attentional allocation towards specific information appearing at different locations, while the second is based on the competition of attentional resources between two different types of information that are co-occurring in the exact location of space. The former is referred to as the "attentional probe task", wherein two stimuli (e.g., threat-related and neutral) are typically presented simultaneously on a computer screen. The participant's task is to respond to a probe stimulus that appears in one of the stimulus positions shortly after the two stimuli have disappeared. A fast reaction time suggests that attention has been directed at the stimulus in the position of the probe, whereas a slow 
reaction time suggests that attention has been drawn away from the stimulus in the position of the probe. The latter attentional task is referred to as the "emotional Stroop task". Because the present thesis employed the emotional Stroop task as a measure of selective attention, it will therefore be discussed in greater detail below.

The emotional Stroop task has frequently been used to examine this kind of cognitive bias. In this task participants are presented a series of words of differing emotional valence, printed in a variety of colours. They are instructed to name the colour as quickly as possible, while ignoring the meaning of the word. It has been proposed (Mathews, 1990) that all words are automatically processed for meaning, but when the word has a meaning that is closely related to an individual's current concern, the individual will have difficultly in ignoring the meaning of the word and will therefore be slower to name the colour of these words compared to neutral words; this is referred to as the Stroop interference effect. In particular, these effects appear to be strongest when the words match their particular fear (Williams, Mathews, \& MacLeod, 1996). The presentation format of the stimulus content in the emotional Stroop task have also typically been of two kinds: One is the "card" version, by which all stimulus words from one valence category (e.g., threat- related) are presented simultaneously on a single card, and the time spent colour naming all words on the card is taken as a latency measure of each word category. The second is the "single trial" computerised version, by which single words are presented one at a time with a short interval between each trial, and each single trial's colour naming latency is recorded. Two studies (Kindt, Bierman, \& Brosschot, 1996, 1997) comparing these formats found that the convergent validity between them was very low. For obvious reasons, the lack of convergence between the formats complicates matters when one wishes to articulate hypotheses as to the underlying mechanisms of selective attention. The difference between the two formats has been discussed in terms of (1) priming effects, in the sense that blocked presentation (compared to single-trial) facilitates inter-stimuli priming (Dalgleish, 1995); (2) blocked presentation is more likely to be affected by conscious strategies; and (3) the blocked format effects may occur at a more superficial level of processing (Kindt, Bierman, \& Brosschot, 1996).

The Stroop interference effect has, however, been subjected to alternative interpretations. One is concerned with whether prolonged colour naming latencies for threat-related words relative to neutral ones in fact reflect a process of cognitive avoidance rather than a product of attentional resources being consumed (de Ruiter $\&$ Brosschot, 1994). However, empirical evidence to support this avoidance hypothesis is presently scarce. Taken as supportive of the selective attention view, first, spider fearful participants' scores on a monitoring scale in a behavioural approach test were positively correlated with Stroop interference (Chen et al., 1996). Second, in a study by Hafer (2002), all of the participants viewed a scene in which an innocent victim was being assaulted. There were two conditions in the design: a "retribution condition" wherein the assailant was caught and was punished, and a "no-retribution condition" wherein the assailant fled and was never punished. The 
main finding was that participants in the no-retribution condition exhibited greater Stroop interference for justice-related words than did their counterparts in the retribution condition. Consequently, it is difficult to reconcile with the proposal that Stroop interference is caused by active avoidance of emotional material, unless one is willing to argue that the Stroop interference effect is caused by active avoidance of justice-related words after viewing an innocent victim being assaulted. Third, if Stroop interference is caused by active avoidance of threatening material, why are in fact some individuals measurably faster to colour name threat words compared to neutral control words, showing the Stroop facilitation effect? The possibly most plausible explanation for some individuals being faster to colour name threat words than neutral ones is that these individuals defend against threatening material in order to avoid being emotionally affected by its content. Although the focus thus far has been on the selective attention towards threatening material, and its possible role in the development and maintenance of anxiety disorders, the selective attentional avoidance of threatening information will be discussed in greater details in later in the Introduction.

Another criticism that has been directed at the emotional Stroop task concerns the possibility that the interference effect could be due to threat-related words' likelihood to have a higher frequency usage among high anxious people. That is to say, the interference effect should arise simply because anxious individuals are experts at processing threat-related material, rather than the difference in emotional valence of the presented material is being responsible for this effect (e.g., Bradley, Mogg, Falla, \& Hamilton, 1998). However, there is evidence showing that the Stroop interference effect seen in clinically anxious participants prior to treatment is abolished shortly after the termination of cognitive therapy treatment (e.g., Mogg, Bradley, Millar, \& White, 1995). This is inconsistent with the frequency-based interference effect, as this would not be expected to change following treatment intervention in which patients become more expert at processing information related to their psychopathology.

One of the most puzzling aspects of the research into selective attention is the lack of convergent validity between the two measures of selective attention (i.e., the emotional Stroop and the attentional probe), despite the relative consistency of the findings. For example, compared to controls, patients with social phobia show selective attention to social threat words on both the emotional Stroop task (Mattia, Heimberg \& Hope, 1993) and the attentional probe task (Musa, Lépine, Clark, Mansell, \& Ehlers, 2003), but that equivalent effects have also been achieved by means of the two different attentional tasks by using the same participants (e.g., Mogg et al., 2000a). The inter-task associations between bias indices are either moderate (Egloff \& Hock, 2003), small (Brosschot, de Ruiter, \& Kindt, 1999) or nonexistent (Mogg et al., 2000a) in effect size which thus implicates that these two measures selective attention seem to be somewhat independent of each other, and perhaps that different underlying processes might be involved. One limitation of the attentional probe task is that it permits several shifts of attention toward and away 
from the threat stimulus before probe onset (e.g., Mogg, Bradley, Miles, \& Dixon, 2004). A reason for the lack of convergent validity suggested by some researchers (e.g., Brosschot, de Ruiter, \& Kindt, 1999) is the different stages of information processing. That is, the attentional probe task is assumed to capture effects occurring at a later stage of processing compared to the effects seen in the emotional Stroop task. Another reason for the lack of convergent validity that has been put forward is that there are in fact different processes involved. To be precise, whereas the attentional probe task is assumed to reflect the process of visual scanning for cues, the emotional Stroop task is assumed to reflect a process in which specific information must be disentangled (e.g., Mogg et al., 2000a). Even though the lack of inter-task association points to the involvement of different underlying processes in selective attention, it does, however, seem reasonable to assume that a common basic underlying mechanism must indeed be involved in order to account for the fact that corresponding effects can be achieved by means of these two different measures of selective attention.

\section{Selective attention: Conscious or an unconscious phenomenon?}

Some recent studies have focused on the issue of whether or not attentional processes are under conscious control. One way to answer this question has been to use subliminal presentation of the stimulus material. The essential idea is that if selective attention occurs without conscious awareness of the stimuli, it must be a preconscious phenomenon. Indeed, several studies have found that individuals with various anxiety disorders selectively attend to threat-related material. For example, using the emotional Stroop methodology, selective attention to subliminally presented threat words has been found in generalised anxiety disorder for threatrelated words (e.g., Mogg et al., 1995; Mogg, Bradley, Williams, \& Mathews, 1993), in spider phobics for spider-related words (Van den Hout, Tenney, Huygens, \& de Jong, 1997), and in panic disorder for panic-related words (Lundh, Wikström, Westerlund, \& Öst, 1999). Perhaps even more noteworthy, this effect is by no means constrained to clinical populations. To be precise, there is evidence that high levels of trait anxiety are associated with selective attention towards threat-related material in conditions in which the words cannot be consciously perceived (e.g., MacLeod \& Rutherford, 1992; van Honk et al., 2001).

A well-replicated finding is that Stroop interference bias scores for masked and unmasked words do not correlate, and therefore seem to measure different kinds of processes (e.g., Lundh, Wikström, \& Westerlund, 2001). It is further notable that laterality effects in selective attention to threat have been observed. Specifically, using the attentional probe task to assess allocation toward threatening faces, Mogg and Bradley (1999) found preattentive bias toward threatening faces in two experiments, an effect that in turn tended to be stronger among high anxious participants in only one of the experiments. In that study, this subliminal effect was predominantly apparent when threat and probe stimuli appeared on the left side of 
the display, hence, indicating greater right hemispheric involvement. These authors suggested that the right hemisphere largely mediates two separate cognitive functions: unconscious discrimination of threat, and the subsequent orienting towards its location. Interestingly, using neuroimaging, Morris, Öhman and Dolan (1998) found evidence of enhanced neural response in the right amygdala (but not in the left) to subliminal (i.e., masked) presentation of aversively conditioned angry faces. In contrast, when the same angry face was available for conscious awareness (i.e., unmasked), there was an enhanced neural response in the left amygdala (but not in the right). These findings suggest that the response to an aversive stimulus is lateralised according to the participants' level of awareness.

The subliminal presentation of the stimulus material can, of course, be subjected to some critique. In particular, it is indeed an artificial approach to elucidating how unconscious processes affect behaviour, and we in daily life rarely have such limited time to inspect a stimulus. However, it should be stressed that the information to be perceived by no means has to be "subliminal" in order to influence behaviour without us humans being aware of it. In fact, research has provided evidence that information presented outside the focus of attention (supraliminal exposure) worked equally well in order to produce qualitatively equal effects as in the case of subliminal presentation of the stimuli being used (Merikle \& Joordens, 1997).

The relative contribution of unconscious processes on behaviour offers perhaps one of the most intriguing and challenging questions of modern psychological research. The question of how, when, and to what extent our behaviour is affected by something that is not available to conscious awareness has been intensely debated (e.g., Greenwald, 1992; Lewicki, Hill, \& Czyzewska, 1992; Loftus \& Klinger, 1992). However, this is an area that has sparked increased interest in conducting experimental research among psychologists from both the cognitive and psychodynamic schools in recent years. One important question is whether subliminal stimulation affects complex emotion-linked behaviour. Another important question is whether effects of subliminal stimulation are relatively brief or somewhat persistent. As an example, based on the somewhat controversial research paradigm of subliminal psychodynamic activation (e.g., Silverman, Lachman, \& Milich, 1982), a recent study showed that the effects are quite persistent (Sohlberg \& Birgegard, 2003). These results were based on a series of studies examining the effects following subliminal stimulation with the phrases "Mommy and I are One" and "Mommy and I are Dissimilar". In this study, compared to the neutral control stimuli, effects of unconscious stimulation with these phrases were observed ten days later. Specifically, relatively strong correlations between various variables (e.g., secure attachment and depression) were consistently observed in the experimental condition, whereas in the control condition the same correlations often approached zero. According to these authors, "such complex persistent effects may follow when powerfully activating stimuli administered under wholly unconscious conditions provokes schematic processing of social information and behavioral confirmation" (pp. 302). 
A further important question is whether unconscious processes have relevance for the aetiology of psychopathology. Bearing relevance to this, Öhman and co-workers (e.g., Öhman \& Mineka, 2001, for a review) have focused extensively on skin conductance responses while convincingly demonstrating that emotional responses can be elicited through perception of environmental threat cues occurring outside conscious awareness. In addition, fear conditioning can occur to fear-relevant stimuli, despite conditioned stimuli being masked to prevent conscious identification. According to Öhman (1996), this kind of preattentive processing of threat is a contributing factor in the development of anxiety disorders, as it can lead to a surplus of autonomic information that has no obvious origin (i.e., not consciously represented). Based on the conclusion drawn from a large number of studies, Öhman and Mineka (2001) proposed the existence of a module, evolved for fear elicitation and fear learning, which they labelled the fear module. The fear module is assumed to be an evolutionarily shaped and relatively independent mental, behavioural and neural system that contains four main characteristics. According to the authors, the fear module is (1) relatively selective with regard to the input to which is responds, but also sensitive to stimuli that have been associated with threatening situations in the evolutionary past, (2) therefore automatically activated by such stimuli, and (3) once activated, relatively impenetrable to cognitive control, thus encapsulated. Finally, the amygdala seems to be the central brain area dedicated to the module (4), that is, there is a specific neural circuitry associated with the fear module. The discussions regarding differences in emotional reactions to fearful versus non-fearful stimuli fits nicely with such neurobiological findings showing the existence of two different pathways (cortical and subcortical) contributing to the emotional processing of information, with the amygdala playing a central role (LeDoux, 1994). The subcortical pathway operates in a "quick and dirty" fashion in the sense that information bypasses the thalamo-cortical and the cortical-amygdala pathway. When stimuli are relayed directly from the thalamus to the amygdala, an immediately initiated fear response is facilitated (quick) but the absence of input to the cortex also implicates that emotional stimuli are not fully analysed (dirty) before action is taken. The thalamo-amygdala pathway has been further suggested to have a survival value in dangerous situations that call for immediate.

The general impression from a large and growing body of evidence casts no doubt that the emotional meaning of information presented outside conscious awareness can influence subsequent responses in a congruent direction (see also, for example, Bargh \& Ferguson, 2000). As will be emphasised at the end of the Introduction, the selectivity of unconscious attentional processes may indeed play a contributing role in the aetiology of anxiety disorders. 


\section{Mechanisms underlying selective attention towards threat}

Until now, many of the topics presented here have been constrained to instances in which selective attention occurs, thus, to type of person and state of mood. But how is bias to be best understood and explained? To gain a more complete depiction of this phenomenon, we need to take a closer look at the information processing systems that might be accountable for this phenomenon. The common assumption among researchers is that in attentional tasks, such as the emotional Stroop, the stimuli are automatically processed for meaning, and the effects arise because attentional resources are consumed. Perhaps the most widespread explanatory frameworks have been Beck's schema theory (e.g., Beck \& Clark, 1997) and Bower's network theory (Bower, 1981). According to Bower (1981), concepts, emotions, and experiences are represented in the brain by semantic associative networks of interconnected nodes. Every node, when activated, causes automatic and rapid spread of activation through the entire network. Because network representations are associatively connected, and because these networks have become larger and more frequently used in anxious individuals, merely a few matches may be sufficient to cause a massive spread of activation in the memory structure. According to Beck (e.g., Beck \& Clark, 1997), it is the dysfunctional nature of cognitive schemata in anxiety that directs information processing in favour of early detection of incorrectly presumed threat. Emotional material is assumed to draw excessively more processing resources due to the activation of specific schemas that correspond to personal threats. On the basis of accumulating data from this research branch, a number of theoretical accounts for the underlying mechanisms have been put forward. Three of these will be presented briefly in the following section. These were motivated by the fact that (1) they are some recent prominent models that attempt to explain the underlying mechanisms of selective attention, that in addition (2) share some common features, and (3) were deemed more applicable here because the emphasis in this thesis is on unconscious perception of emotional material.

In a modified model that originated from their model formulated a decade earlier, and in contrast to their earlier version, Williams and co-workers (1997) acknowledged that automatic selective attention towards cues reflects a vulnerability factor for clinical anxiety. Their model proposes two separate preattentive processing stages. At the first stage, the threat value of environmental stimuli is assessed by what they labelled as "affective decision mechanism". In the earlier version, if the threat value is sufficiently high, it should trigger the second preattentive processing stage. However, because this version of the model did not include the vital role of competition of attentional resources, this mechanism simply implies that highly threatening cues should be processed more efficiently than non-threatening cues. Anxiety, on the other hand, is characterised by attentional priority being given to threat cues in preference to other cues. In their modified version, the affective decision mechanism was modelled on some thing reminiscent of a parallel 
distribution network (Cohen, Dunbar, \& McClelland, 1990), wherein activation of input units that are "emotionally tagged" (as a consequence of biological preparedness or learned symbolic meaning) are given priority in preference to other competing stimulus units within the same network. The main function of the input units is to assess the threat value of environmental stimuli. If given priority in competition with other units, this would increase the chance to trigger the second preattentive processing stage, which they initially labelled as "resource allocation mechanism, but is now the Task Demand Unit (TDU), in parallel distribution network terms. Thus, increased activation of the input units (due to, for example, the threat value of the stimuli and/or state anxiety) will affect the TDU so that more processing resources are directed towards or away from threat. These authors specifically proposed that high trait anxiety reflects a permanent tendency to respond to increased activation of threat units at the preattentive stage of processing which, in turn, leads to more processing resources being directed towards threat, whereas the opposite is true for low levels of trait anxiety - they should show a permanent tendency to direct processing resources away from threat. In addition, the directions of effect will become more evident when these individuals are simultaneously affected by an increase in temporary state of anxious mood - a pattern that implicates an interaction between trait and state anxiety. It is further assumed that an increase in anxious mood should produce an effect that equals the effect that will occur when the negative valence of the presented stimuli is increased.

The model by Mathews and Mackintosh (1998) is similar to the one described above in that it accounts for the critical role of competition of attentional recourses and that attentional processes are operating prior to conscious awareness, but also in the importance of anxiety levels. According to this model, biased attentional processing favouring threat arises from increased activation afforded by a threat evaluation system (TES) to stimuli associated with danger in anxious individuals. That is, the activation of the representations associated with potential danger is increased by input from the TES (which corresponds to the activation in the input units in the model by Williams and co-workers, as described above). In their view, all perceptual input is automatically assessed for its affective significance, and when it matches stored attributes that have been previously associated with aversive events (as a consequence of biological preparedness, conditioning or symbolic processes), it will receive attentional priority. They further proposed that the activation from the TES is greater in highly trait-anxious individuals, because these individuals (1) have permanently lower TES output thresholds, which should be further strengthened by increases in anxious state, and (2) possess a wider range of threat representations in the TES. In contrast to the model by Williams and co-workers (1997), this model does not assume that attentional resources will always be directed away from threat in low trait-anxious individuals. According to this model, severe threat cues should always attract attention, even in the absence of anxiety, and weak threat cues only attract attention in highly anxious individuals. In their view, highly threatening cues should always be given attentional priority at the expense of competing representations, whereas controlled effort serves to oppose the tendency for weak 
threat cues to become dominant. In fact, one serious problem with the model by Williams and co-workers (1997) is the demonstration of the existence of biased attentional processing favouring highly threatening stimuli irrespectively of anxiety levels (Koster, Verschuere, Crombez, \& van Damme, 2005; Mogg et al., 2000b; see also Wilson \& MacLeod, 2003, for similar finding). In fact, similar to Mathews and Mackintosh (1998), the model by Mogg and Bradley (1998) explicitly proposes that, if the threat value is sufficiently high all individuals should show selective attention. In order to account for this proposal, these authors argued that trait anxiety reflects the reactivity of a valence evaluation system to aversive stimuli. That is, in some individuals rather trivial stimuli are tagged with a relatively high subjective threat value, and therefore, individual differences in anxiety seems more likely to become evident only when an individual is confronted with mildly threatening information, such as words, or mildly naturalistic information (pictorial stimuli portraying mild threat scenes). Put differently, selective attention to relatively weak threat cues is assumed to be a result of bias in the operation of the valence evaluation system in highly trait-anxious individuals, which is further assumed to be the primary factor responsible for mediating vulnerability to emotional disorders.

\section{At times, people selectively attend away from threatening information}

Although the topics have been largely restricted to preferential attentional processing of threatening material, after reviewing research findings, my own view is that perhaps the most remarkable feature of the research on selective attention to threatening information are findings indicating that some participants are in fact measurably faster to colour name threat words than neutral control words (the Stroop facilitation effect). To date, the nature of this anxiety-related bias is poorly understood. Arguably, none of the three models described above provide a detailed analysis of the processes that underlie selective attention away from threat cues. Thus, exactly why the Stroop facilitation effect of threat words arises and the underlying dynamics of this effect are issues yet to be clarified. It has been proposed that low anxious individuals may use controlled effort that serves to oppose the tendency of weak threat cues to become dominant (Mathews \& Mackintosh, 1998). This does in fact explain why the Stroop interference effect should be eliminated. It does not, however, explain the presence of Stroop facilitation (i.e., selective avoidance). Another similar proposal is that for some individuals, conscious strategies might operate in order to neutralize or counteract threat-related information and that a bias for these words will occur only when they are presented subliminally (e.g., MacLeod \& Hagan, 1992; Williams et al., 1996). In contrast to this proposal, despite the fact that the information was not available for conscious processing, there are studies demonstrating that, in a non-clinical sample, some individuals do indeed show Stroop facilitation (Lundh et al., 2001; MacLeod \& Rutherford, 1992). Even though the model by Williams and co-workers (1997) proposes that low trait-anxious individuals will show an increased tendency to direct 
attention away from threat cues as their levels of state anxiety increase (already at a preattentive level of processing), it does not explain the processes that underlie this effect. For example, when attention is directed away from threatening information, this means faster colour naming of threat words compared to neutral ones in the Stroop paradigm. Does this in fact mean that threat words are given less priority in competition with other units, than is the case for neutral words? If so, why is this the case? It has been proposed that attention may be directed away from threatening information when it is evaluated as being mildly aversive and having low threat value, as a function of mood regulation (Mogg \& Bradley, 1998) and thereby this model provided an account for the functional value of attentional avoidance.

Although the discussion thus far points to the fact that anxiety levels are associated with selective attention to threatening information, Eysenck's (1997) theory concerning cognitive bias holds that biased processing may also be mediated by another factor, namely defensiveness. As a matter of fact, Eysenck explicitly proposed that "repressors", or individuals with a repressive coping style (i.e., people who score low on trait anxiety and high on social desirability - the latter being interpreted as defensiveness), have a cognitive bias opposed to the processing of threat. As a consequence of the growing body of research findings involving these individuals, a more complete discussion regarding previous findings will be given below.

\section{Repressive coping style}

According to Weinberger, Schwartz and Davidson (1979), scores on social desirability (e.g., MCSD: Crowne \& Marlowe, 1964) are appropriate for splitting low and high trait anxiety groups into four groups: a) low trait-anxious individuals with low social desirability scores ("true" low trait-anxious); b) low trait-anxious individuals with high social desirability scores (repressors); c) high-trait-anxious individuals with low social desirability scores (high-trait-anxious); d) high-traitanxious individuals with high social desirability scores (defensive high-traitanxious). The major conceptualisation of repressors is of persons who actively avoid negative affect. For example, studies have shown that repressors show a discrepancy between self-reported and physiological indications of arousal (e.g., Weinberger, 1990, for a review), an underestimation of self-reported anxiety compared to observers' ratings (e.g., Derakshan \& Eysenck, 1997, for a review), and difficulties recalling affective events (e.g., Davis \& Schwartz, 1987). Most importantly in the present context, according to Eysenck (1997), high trait-anxious individuals should show cognitive biases for threat information, whereas repressors should show the opposite pattern. The typical research finding that repressors show a discrepancy between their subjective experience of anxiety compared to behavioural as well as physiological indications of anxiety (see Weinberger, 1990, for a review) validates the construct of repressive coping but, of course, the discordance response may be due to increased objective experience of anxiety that does not correspond to the subjective 
experience, or the reversed. Thus, because the repressors have been conceptualised as persons who actively avoid perceiving themselves as anxious individuals (e.g., Weinberger, 1990), the perceived threat to the self (or self-esteem) might trigger physiological responses, whereas the repressors consistently report low subjective experience of anxiety, or perhaps because they are simply unaware of their affective state. Alternatively, the increased attention to internal affective cues might make them even more inclined to report low affect. The general impression gathered from a theoretical paper based on a large body of research (Derakshan \& Eysenck, 1997) is that discordance responses among repressors arise because these individuals selectively avoid attending to their own physiological activity.

With regard to the emotional Stroop task, however, the evidence is far from consistent. One study (Mogg et al., 2000a), in accordance with Eysenck's theory (1997), found a Stroop facilitation effect for threat words in participants with a repressive coping style. Two other studies (Myers \& McKenna, 1996; Newman \& McKinney, 2002) found that all groups except the repressor group showed Stroop interference for threat words vs. control words; the repressor group did not show a facilitation effect, however, but was instead relatively unaffected by the content of threat words. Two other studies failed to find any differential effect in repressors vs. non-repressors (Brosschot et al., 1999; Fox, 1994). Finally, an early study (Dawkins \& Furnham, 1989) found that repressors showed more interference for emotional words than did both low trait-anxious and high trait-anxious individuals. Possible explanations for these divergent results that have been discussed refer to different formats for administering the words (i.e., card vs. single-trial), different categories of threat words (e.g., social vs. physical), and methodological artefacts. For example, Dawkins and Furnham (1989) administered their words using a card format with all words from each category presented on a separate card. If Stroop facilitation in a single-trial format (i.e., with only word exposed at a time) is the result of a strategy to remove threatening words as quickly as possible from the computer screen, and thereby from the perceptual field, this strategy will simply not work in card format (because the card does not disappear from view until all the words on it have been colour-named). Another possibility for the inconsistencies between the findings reported above is the fact that only supraliminal presentation of words was used in these earlier studies. Thus far, to my knowledge, there appear to be no published papers that have discussed the possible effects of subliminal presentation. Because the supraliminal condition has been suggested to be somewhat shaky with respect to stability (e.g., MacLeod \& Hagan, 1992), conscious strategic processes among repressors may serve to override the influence of emotionally threatening words and may cancel out any interference effect. In fact, Calvo and Eysenck (2000), in another type of task, found that repressors had an early bias towards threat, but a late avoidance of threat, which they interpreted as a result of a strategic inhibitory bias. If it is true that conscious strategic processes among repressors may serve to selectively inhibit emotionally threatening words, this should be manifested in Stroop facilitation. With regard to words that cannot be consciously perceived, repressors would be unable to avoid the processing of masked threat words and would 
therefore be expected to behave like high trait-anxious individuals in the masked exposure condition and show Stroop interference for masked threat words. On the other hand, it is also possible that their defensive avoidance of threatening information has become automatic to such a degree that they selectively inhibit the processing of threat already at the preattentive level. A third possibility is that, if conscious strategic processes among repressors may serve to override any possibility of being influenced by emotionally threatening information, one would expect these individuals to be equally fast to colour name threat-related words compared to neutral ones, irrespectively of whether the words are masked or unmasked (Williams et al., 1996). Therefore, faster colour naming latencies in general might indicate whether "non-selective" inhibition of the stimulus content occurs. In various sorts of attentional bias paradigms, however, the general sense is that we, unfortunately, obtain a good amount of method variance across laboratories, which may account for a fair proportion of the variability in findings. For example, variations in the preexperimental information given to the participants are likely to make the conscious strategic processes highly variable from one context to another.

The repressors' use of an avoidant style of dealing with threatening information has further led to suggestions that these individuals may be at risk for serious physical health-related problems, such as cancer (e.g., Schwartz, 1990, for a review). Thus, an avoidant style of information processing would presumably lead to a dissociation of emotional systems so that the system's ability to self-regulate via feedback would be impaired, which is why repressors should be prone to suffering from physical disorders. In contrast, in a study examining individual differences in resting electroencephalographic asymmetry in anterior regions of the brain, Tomarken and Davidson (1994) demonstrated that individuals with repressive coping styles have relative left anterior activation compared to the low and high trait-anxious groups. The importance of this finding is that relative left anterior hypoactivation has previously been found to be linked to increased negative affect (e.g., Sutton \& Davidson, 2000, for a review). This association ultimately led Tomarken and Davidson (1994) to conclude that relative left anterior activation may promote heightened access to cognitive and other regulatory processes that may serve to inhibit the experience of negative affect and to sustain positive affect. In other words, relative left anterior activation may index decreased risk for psychopathology. Consistent with this reasoning, higher scores on social desirability have been found to be associated with a decreased lifetime prevalence of affective disorders and other manifestations of psychopathology (Lane, Merikangas, Schwartz, Huang, \& Prusoff, 1990). Thus, this clearly suggests that there may be some beneficial features associated with defensiveness/repressive coping, despite claims suggesting that repressive coping is associated with behaviours that may implicate negative consequences with regard to health issues. As will be discussed below, there are also some beneficial features associated with attentional avoidance of threatening material. 
After a review of the literature concerning repressive/defensive coping, the general impression is that it is somewhat fraught with methodological inconsistencies and inferential ambiguities, for example, the absence of a non-arbitrary standard for what constitutes a "true" repressor. The current practice in many studies is to select subjects from a sizable pool based on extreme scores, and then routinely divide groups based on median splits, or in some cases third splits. Furthermore, many studies have traditionally used only the high anxious/low social desirability, low anxious/low social desirability and low anxious/high social desirability (repressor) groups in their design, thereby neglecting the high anxious/high social desirability group. As, a consequence, any result for the repressor group could simply be due to high social desirability scores, and at the same time, it is unclear whether any result obtained in the high anxious/low social desirability group also holds for the high anxious/high social desirability group. Furthermore, the somewhat widespread use of the repressor vs. non-repressor division as a between-group factor arguably exacerbates these problems even further. In particular, what is somewhat striking is that conclusions drawn for the low-defensive or non-repressor groups also concern the high trait-anxious group. These individual are, for example, more likely to show selective attention to threat cues, which is further assumed to be a vulnerability factor that predisposes to anxiety disorders (e.g., Eysenck, 1997; Williams et al., 1997). Furthermore, high trait-anxious participants' responses have been found to be significantly lower (compared to low trait-anxious) on measures of autonomic reactivity (e.g., Jezova, Makatsori, Duncko, Moncek, \& Jakubek, 2004; Wilken, Smith, Tola, \& Mann, 2000) when confronted with stressors. The diminished autonomic reactivity in response to stressful events has, in turn, been interpreted as maladaptive responses (e.g., Hoehn-Saric \& McLeod, 2000). Consequently, it is therefore probably erroneous to conclude that, for example, "stress and behavioral approach may stand in a negative feedback loop relationship in low defensive individuals" (Kline, KnappKline, Schwartz, \& Russek, 2001, pp. 1141).

\section{What is the functional significance of selective attention away from negative information?}

In order achieve a better understanding of the underlying dynamics of processing of threat, one should perhaps address this issue differently by asking the question "what is the functional value of diverting attention away from threat?" Even though the discussion thus far has been restricted to negative consequences for the individual, there are positive features of anxiety. Anxiety acts as a warning signal for future danger or harm, and is therefore adaptive. Some authors have argued that avoidance of threat is not always adaptive (e.g., Mathews \& MacLeod, 2002; Mogg et al., 2000b). From an evolutionary perspective, we must have an effective threat detection system so that attention is directed toward severe (but not to mild) threat in our environment. At the same time, avoidance of some stimuli might indeed have adaptive functions for us. For instance, we can continue to focus on current goalrelevant activities instead of being disrupted by irrelevant information, but it may 
also play a useful role in mood regulation, assisting us to maintain a positive state of mood (e.g., Mathews, 1990).

In a section above, some recent theoretical accounts for the underlying mechanisms of selective attention towards threatening attention were summarised briefly. There now seems to be a broad general agreement that strong threat cues should always attract attention, but weak cues only do so in highly anxious individuals. Curiously, even though much attention has been directed to the notion that non-anxious individuals will show selective attention to threatening material, provided that the threat value is sufficiently high, little effort has been made to explain the fact that anxious individuals occasionally show selective attention away from naturalistic threat stimuli at a relatively early stage of information processing. For example, social anxiety has been found to be associated with selective attention away from briefly presented $(500 \mathrm{~ms})$ pictures portraying negative facial expressions as a function of increased anxious state (Mansell, Clark, Ehlers, \& Chen, 1999), and higher levels of social anxiety and trait anxiety are associated with Stroop facilitation for masked (i.e., subliminal) angry faces (Putman, Hermans, \& van Honk, 2004). To be in agreement with the proposition that high anxious individuals subjectively evaluate the threat value of a stimulus as being disproportionately high (Mogg \& Bradley, 1998), the critical point that must be made is that, logically, any particular threat cue must have higher threat value for an anxious individual than for his non-anxious counterpart and, accordingly, should be associated with selective attention towards threat.

When accounting for the underlying mechanisms of selective attention, one must be able to account for selective attention towards threat, as well as selective attention away from threat. Of course, the stimuli still must be evaluated by content in order to trigger actions to avert real danger, or even to avert minor threat. That is to say, the threatening information is not ignored in the sense that the information was never fully processed by content. Thus, on the basis of humans having an effective threat detection system, processing priority is always given to threatening information regardless of current anxiety status. By quickly (unconsciously) evaluating the threat value of stimuli, the function of the detection system is to operate in a manner that could be conceptualised as a naturally occurring processing bias (or a "default mode") that, in the case of real danger, prompts for immediate and appropriate action in a subsequent stage of information processing. Avoidance would be an appropriate action if real danger appears, and prolonged attention towards real danger would therefore be highly dysfunctional. In the case of relatively harmless threats such as, threat cues presented in a laboratory, attentional avoidance without conscious awareness of the perceived information would be useful because it assists us in focusing on current goal-relevant activities as well as in maintaining a positive state of mood. Prolonged attention directed towards the very same threat, on the other hand, has the exact opposite effects and, hence, would be highly dysfunctional. This does capture an important aspect of stages in information processing that implies that the malfunction in an information-processing system is not reflected in 
the initial stage, but rather in the following stage which, in turn, is likely to continue to play an important role in anxiety. If this argument is plausible, we need to take a look at studies that have specifically investigated separate effects of engagement (i.e., detection speed) to and disengagement (i.e., shift in attention) of threat. A review of contemporary research on attentional allocation toward threat cues did indeed show that the effect of biased attentional processing is a result of the participants having difficulties in disengaging their attention from threat rather than experiencing speeded detection of threat cues. To be precise, difficulty in disengaging attention from threatening cues has been found in social phobia for social threat words (Amir, Elias, Klumpp, \& Przeworski, 2003), in high trait-anxious individuals for angry faces (Fox, Russo, \& Dutton, 2002) and threatening pictures (Yiend \& Mathews, 2001), and in high state anxious individuals for angry faces and threat-related words (Fox, Russo, Bowles, \& Dutton, 2001). Also noteworthy, none of these studies found any evidence supporting the notion of anxiety being associated with facilitated detection of threat-related cues.

The reasoning above is in agreement with findings showing that children, irrespective of clinical status, show biased processing favouring threat-related information (Kindt \& Van den Hout, 2001: Kindt, Van den Hout, de Jong, \& Hoekstra, 2000). Based on their research findings from child samples, Kindt and coworkers also suggested that adequate inhibition of threat cues must be learned during the course of development, and that childhood anxiety may prevent adequate inhibition from being learned. These authors concluded that inadequate inhibition of threatening information that is selectively attended is the factor accountable for the development of clinical anxiety. The inadequate inhibition explanation also fits nicely with how the Stroop interference effect can be interpreted. Because participants are explicitly instructed to ignore the content of the words, the pronounced failure to inhibit the meaning of the words when their nature is threatening rather than neutral could in fact be the factor responsible for the Stroop interference effect. If negatively valenced material is not inhibited, following Bower's (1981) network model, a rapid spread of activation through the entire network will occur and, in addition, will interfere with other cognitive tasks at hand. In anxiety and worry, Thayer and Friedman (2002) strongly emphasise the role of inhibition in efficient and flexible functioning.

\section{Why study selective attention in non-clinical populations, and why use the emotional Stroop task?}

As noted earlier, selective attention towards negative material has been proposed as a factor in the development of clinical anxiety. That is, numerous studies have established that various anxiety disorders are accompanied by the tendency to attend to fear-relevant stimuli, even if they are irrelevant to the task the participants are performing (Williams et al., 1996, for a review). At the same time, a number of researchers have asserted that this bias is not the nonessential side effect of 
pathological anxiety, but plays a crucial role in the maintenance of this form of psychopathology (e.g., Beck \& Clark, 1997; Eysenck, 1997). In order to clarify whether selective attention play a significant role in the maintenance of anxiety disorders, the researcher must establish if (1) anxiety promotes selective attention and (2) selective attention in turn promotes anxiety. The available evidence to date supporting the maintenance hypothesis seems compelling. In answer to the former, as already reviewed, elevated enduring anxiety levels (in clinical and non-clinical individuals) are associated with selective attention, and are likely to be further strengthened by increased stress. If, in addition, increased anxiety leads to intensification of selective attention, the reverse should be true. In answer to the latter, the effect of selective attention to threat cues seen in clinically anxious participants prior to treatment is in fact eliminated shortly after termination of the treatment (Mogg, Bradley, Millar, \& White, 1995; Van den Hout, Tenney, Huygens, \& de Jong, 1997).

\section{Selective attention to unconsciously perceived information as a factor in the development of clinical anxiety}

A previous heading voiced a question concerning the functional significance of selective attention away from negative information. In answer to this question, selective attention towards threatening information may indeed have negative consequences for the human. To date, there are indications from several sources that point to the fact that selective attentional processing of threat-related material unavailable for conscious awareness is indeed associated with emotional distress in response to stressful events. Using the subliminal Stroop interference bias as a predictor, preattentive bias has been found to significantly predict emotional responses to stressful real-life situations (MacLeod \& Hagan, 1992; Verhaak, Smeenk, van Minnen, \& Kraaimaat, 2004; Pury, 2002), as well as emotional responses to somewhat more artificial stressful events (Nay, Thorpe, Roberson-Nay, Hecker, \& Sigmon, 2004; Van den Hout, Tenney, Huygens, Merckelbach, \& Kindt, 1995). Although selective attention to threatening information has been suggested to be a factor in the development and maintenance of anxiety disorders, empirical evidence supporting this view has been absent until very recently. That is, in a series of experiments by MacLeod and co-workers (MacLeod, Rutherford, Campbell, Ebsworthy, \& Holker, 2002; Matthews \& MacLeod, 2002), there was evidence that non-anxious participants who were trained to develop biased attentional processing of threat cues responded with a more negative mood state when exposed to a stressful task than did those who did not receive such training. Biased attentional processing would seemingly lead to the overrepresentation of threat cues within the information that anxious individuals encode from their environment. Accordingly, if people expend more processing resources to the presence of environmental threats, this in turn may increase anxiety further. Put briefly, the rationale is that this bias leads to a heightened sensitivity to threat-relevant material. As a consequence, this material is selectively processed and becomes more easily accessible. This, in turn, increases anxiety levels, which leads to further selective processing of threat cues and so on. 
A limitation of the emotional Stroop task that has been put forward (e.g., Mogg \& Bradley, 1998, 1999) is that in single-word presentation, words have fairly limited threat value. These authors specifically proposed that among low anxious individuals, threat words should have little or no threat value, whereas pictorial stimuli should be more useful for the purpose of assessing biased processing of threat. With regard to the usage of pictorial stimulus material, it is assumed that unconscious processing of threat is mediated by evolutionary-driven mechanisms that, in turn, are more likely to be triggered by pictorial representations of biologically relevant threats (e.g., Öhman, 1996). Nonetheless, what remains to be explained is why (1) individuals with elevated levels of anxiety are more likely than low anxious individuals to show preattentive bias to threatening information, and (2) the same kind of bias is associated with emotional responses to stressful events when word stimuli are used (i.e., mild threat value). This is of paramount importance because, even if threat words could be viewed as not being "potent" threat cues that, in addition, are not assumed to be biologically relevant threats, the individual differences in which threat words are processed are still a potential marker for the development of anxiety disorders. Thus, the underlying dynamics of the mechanisms that determine whether processing favouring mild threat (i.e., threat words) will occur or not are likely to be the key features in the development of clinical anxiety.

\section{The empirical studies}

The aim of the present thesis is threefold. One focus is on the identification of factors that either promote or prevent preattentive bias for threat-related information in nonclinical populations. Given the central role of trait anxiety in this kind of research, but at the same time taking Eysenck's theory into account, the personality factors of interest in the present thesis were trait anxiety and social desirability. If there are personality factors that are frequently involved in determining the response pattern, are these factors independent of or dependent on other factors such as, temporary elevations of anxious state?

The role of unconscious perceptual influence on autonomic responses (as exemplified in the work of Öhman and co-workers, mentioned above) has an apparent relationship to research on selective attention. Because biased processing of potentially threatening external cues play a central role in the definition of trait anxiety, this could possibly be a critical factor determining the autonomic responses to masked threatening pictures as well. Thus, the second aim of the present study was to examine the roles of trait anxiety and social desirability on autonomic responses elicited by subliminal presentation of threatening pictures.

As described earlier, the way we process information predicts our emotional responses to stressful events, but we may need several sound indicators of emotional response to stressful events. Undoubtedly, stressful events are typically accompanied 
by physiological responses. Even though the relationship between selective attention to threatening material (subliminally presented) and subjective emotional responses to stressors seems to be established, its possible relationship to objective emotional responses to stressors has yet to be clarified. Thus, the third aim was to investigate whether the masked version of the emotional Stroop task (i.e., preattentive bias) could be used to predict the physiological responding (as measured with skin conductance) to a stressor (see Table 1 for an overview of demographics, focal predictors and outcome measures for all studies).

\section{Method}

\section{Instruments}

Karolinska Scales of Personality (KSP). This instrument was developed by Schalling (Schalling, 1985), and consists of 15 subscales. Three of these subscales were used: Somatic Anxiety, Psychic Anxiety, and Social Desirability (each with ten items). Somatic Anxiety refers to autonomic symptoms, concentration difficulties, vague distress, and panic attacks, whereas Psychic Anxiety is intended to represent worrying, anticipatory anxiety, insecurity, and social anxiety. In Study I, the participants' scores on Somatic Anxiety and Psychic Anxiety were summarised into a Trait Anxiety score. The combined KSP trait anxiety scale has been found to correlate strongly with Spielberger's STAI-T (Oldenburg, Lundh, \& Kivistö, 2002). The KSP scales Somatic Anxiety and Psychic Anxiety also correlate highly $(r=.69$ in the present sample). The Social Desirability scale (KSP-SD) is a short form of the SDS (Crowne \& Marlowe, 1964) and includes items dealing with social conformity as well as being friendly and helpful. The KSP scales have demonstrated adequate psychometric properties (Gustavsson, Weinryb, Göransson, Pedersen, \& Åsberg, 1997). The KSP-SD was used in Studies I, III, and IV.

Spielberger's State-Trait Anxiety Inventory (Spielberger, Gorsuch, Lushene, Vagg, \& Jacobs, 1983). The trait version (STAI-T) is a 20-item scale that measures enduring symptoms of anxiety. STAI-T was used in Studies II, III, and IV. The state version (STAI-S) is a 20-item scale that measures current subjective reports of worry and anxiety. STAI-S was used in Studies II and IV.

Impression management (IM). This instrument consists of 12 items assessing social desirability, was selected from the 16PF Fifth Edition Questionnaire (Cattel, Cattel, \& Cattel, and comprises elements of both self-deception and other-deception. This instrument has adequate psychometric properties $(\alpha=.65)$ (Russell \& Karol, 1997), and was strongly correlated $(r[41]=.78)$ with a short form of the MCSD scale (Crowne \& Marlowe, 1964) in a recent pilot study conducted in our laboratory.

Worried mood. In Study III, immediately before the Stroop task participants were asked to complete a set of four visual analogue scales in answer to the question: 
"How do you feel right now?". One of these asked them to indicate their degree of worried mood by a mark on a $100 \mathrm{~mm}$-long line with the left endpoint labelled "not at all" and the right endpoint as "maximum". This scale was strongly correlated with Spielberger's STAI-S $(r[47]=.78)$ in a previous pilot study conducted in our laboratory.

Subjective emotional response (SER). In Study IV, immediately after the problemsolving task (a stress task) participants were asked to complete six visual analogue scales in answer to the question: "How did you feel during the task?" The items that were derived from the STAI-S, paraphrasing some original items, and included statements such as "I felt tense", "I was nervous", and "I was calm" (reversed). Levels of stress were indicated by a mark on a $100 \mathrm{~mm}$-long line with the left endpoint labelled "not at all" and the right endpoint as "maximum". The internal consistency for this scale was satisfactory $(\alpha=.85)$.

\section{Assessment of awareness}

In the studies, subjective reports were used to ascertain that subliminal presentation of stimuli remained unavailable for conscious awareness. A subjective threshold refers to the level of discriminative responding at which observers claim not to be able to identify or recognise perceptual information, but when forced might detect it at a better-than-chance level of performance. The objective threshold is the level of discrimination responding at a chance level of performance (e.g., Cheesman \& Merikle, 1986). Thus, acknowledging Merikle and Reingold's (1992) claim that the employment of an objective threshold method to establish the threshold of conscious perception will possibly eliminate any evidence of unconscious effects, the subjective threshold method was applied across all studies. In all studies in the current thesis, the commonly used method of backward masking for both words and pictorial emotional stimuli to protect against conscious perception of the material was adapted.

The test sessions started with a pre-test to establish an individual threshold for each participant's conscious word or picture identification. In the emotional Stroop task, this test comprised eight neutral words. In each trial, the word was masked in the same manner as described above. Each word was programmed to be exposed first for a period of $17 \mathrm{~ms}$. If a participant could not name a word on the first occasion, the word was presented again, with an exposure duration that increased by increments of $17 \mathrm{~ms}$ until the participant was able to identify the word. The participants were not encouraged to make guesses, nor were they explicitly discouraged from guessing. This procedure was repeated for all eight words with the purpose of establishing individual thresholds for conscious identification for the upcoming subliminal exposure condition. In the Picture Perception task, the threshold task consisted of the presentation of one neutral picture, masked in the same manner as described above. The picture was first exposed for a period of $6 \mathrm{~ms}$, which was the 
fixed exposure duration used in the masked condition. In the ensuing presentations, exposure duration increased by increments of $6 \mathrm{~ms}$ until the participant was able to identify the picture.

\section{Stimulus material and presentation}

Single-trial computerised presentation of the stimuli was used in all studies. In all the emotional Stroop studies, there were 192 trials during the Stroop task. The words were divided into three or four word categories (see below), and were matched with regard to word length and frequency. The words in each category were subdivided into two sets of words each, which were also matched in the same respects. One of these word sets was presented supraliminally (unmasked) during the Stroop task whereas the other set was presented subliminally (masked) during the Stroop task. Each of the stimulus words in each exposure condition was shown four times, once for each colour: red, green, blue and yellow. Exposure conditions, colours and words were presented in a fully randomised fashion across the 192 trials.

As Study I used data that were a part of a larger project, focused primarily on questions concerning health and illness (Lundh et al., 2001), illness-related threat words used principally to test selective attention to threat cues but also negative emotion words (negatively valenced words from different categories) were used. In Study II pictorial stimuli were employed. Pictures of affective events and objects are assumed to be ecologically valid stimuli in the sense that visual information is often the first cue signalling threat in the natural environment (e.g., Öhman, 1997). Fourteen pictures were taken from the International Affective Picture System (Lang, Bradley, \& Cuthbert, 1999), chosen on the basis of their normative pleasure and arousal ratings. There were seven unpleasant and arousing pictures, mainly of a physical threat nature (e.g., mutilated bodies, battered woman). There were also seven pleasant and calm pictures, differing in nature (e.g., book, umbrella). The pictures were presented both masked and unmasked (i.e., 28 trials). In Study III, in accordance with some previous studies (e.g., Brosschot et al., 1999; Mogg et al., 2000) that have focused on effects of high-defensive low trait-anxious participants (i.e., repressors), social and physical threat words were used. Additionally, selective processing of social threat in particular should be distinguishing factor between repressors and others. Fox (1994), for example, argued that the items on a measure of social desirability (used to identify repressors) focus on social evaluative issues. In Study IV, we believed that ego threat words might be particularly useful in this context. That is, the stress task constituted a problem-solving task that had to be completed within a very limited time; this would facilitate processing biases towards ego-threat interpretations, as feelings of danger to self-esteem and social approval should be activated in some subjects under these conditions. In addition, words labelled as "ego positive" (i.e., the ego threat words were practically "reversed") were included. This was motivated by the fact that some studies employing facial stimuli (e.g., Mogg, Philippot, \& Bradley, 2004) have used emotionally incongruent 
stimuli, such as angry and happy faces. Finally, physical threat words were also included to determine if there was a general or content-specific effect with regard to preattentive processing of threat as a predictor of emotional responses to a stressful event.

In the emotional Stroop task, in the supraliminal exposure condition, a trial involved the presentation of a coloured stimulus word that remained on the screen until the participant's verbal colour naming response triggered the voice key, after which the screen was blanked for $2 \mathrm{~s}$ until the presentation of the next stimulus word. In the subliminal exposure condition, a trial involved the presentation of a letter string of Xs used as a forward mask $(17 \mathrm{~ms})$ followed by a stimulus word shown for the appropriate exposure duration (depending on an individually established threshold, see below). Then, a letter string of Xs used as backward mask replaced the stimulus word and remained on the screen until the participant's verbal colour naming response triggered the voice key, after which the screen was blanked for $2 \mathrm{~s}$ until the presentation of the next trial. The masks presented immediately before and after the stimulus word consisted of an equivalent length of $X_{s}$ and were presented in the same colour. The supraliminal condition was identical for all emotional Stroop studies, but the subliminal condition differed slightly from Studies III and IV. That is, the briefly presented stimulus word was not preceded by a forward mask, as was the case in the other studies.

In the Picture perception task, in the masked exposure condition, a trial involved the presentation of a picture that remained on the screen for $6 \mathrm{~ms}$, immediately followed by a mask (consisting of picture fragments of the original picture) remaining on the screen for another $200 \mathrm{~ms}$. Then, the screen was blank for $16 \mathrm{~s}$ until a white dot appeared on the screen $4 \mathrm{~s}$ prior to the presentation of the next picture. In the unmasked exposure condition, a trial was identical to a trial in the masked condition, except this time the picture remained on the screen for $2 \mathrm{~s}$ before it was masked. There were two different presentation orders (participants being randomised to them): (1) seven neutral followed by seven threatening; (2) seven threatening followed by seven neutral. In all cases, masked presentation of pictures always preceded unmasked presentation.

\section{Measurement of preferential processing}

As dependent variables in three studies, as a measure of selective attention, Stroop interference indices were calculated for each individual by subtracting the mean colour naming latency for neutral words (unmasked, masked) from the mean colour naming latency for threat words (unmasked, masked). A positive value indicates a slower colour naming response for threat words as compared to neutral words (i.e., Stroop interference), whereas a negative value indicates the opposite (i.e., Stroop facilitation). As dependent variables in Study II, targeting selective autonomic responses, threat indices were calculated for each individual by subtracting the mean 
range value (defined as the distance between the lowest and highest skin conductance levels during the measurement period) for neutral pictures (unmasked, masked) from the mean range value for threat pictures. As regards the Stroop index, a positive value indicates an increase in skin conductance responses for threatening pictures relative to neutral pictures, whereas a negative value indicates the opposite.

As an additional task in Study I, after the end of the Stroop task, a tachistoscopic identification task was used to test for implicit memory effects of the words shown during the Stroop task. In this task, 72 words were shown once in a fixed order, at the briefest exposure duration at which the individual could identify any word during the pre-test (see below). Specifically, the 24 words presented supraliminally (unmasked words) and the 24 words presented subliminally (masked words) during the Stroop task, were again presented along with another set of 24 words (a word set served as unprimed words). The 24 words in each mode of presentation (supraliminal exposure, subliminal exposure, or non-exposure) were subdivided into three sets of eight words each (illness-related, negative emotion, and neutral). When performance is compared on exposed vs. non-exposed words, these effects are usually referred to as priming effects (or implicit memory effects). When priming for emotional vs. non-emotional words is compared, these effects are referred to as implicit memory bias effects. Thus, as an additional set of dependent variables in Study I, implicit memory bias, indices for unmasked and masked threat words were calculated for each individual by subtracting the priming of neutral Stroop words (unmasked and masked, respectively) from the priming of threat-related Stroop words (unmasked and masked). A positive value indicates a memory advantage for threat words as compared to neutral words, whereas a negative value indicates the opposite.

\section{Statistical analyses}

Hierarchical multiple regression analyses were used in all the studies included in this thesis. In hierarchical regression, the researcher uses a priori hierarchical structure to build the model, and examines the influence of several predictors in a sequential way. Thus, the focus is on the change in predictability associated with predictors entered later in the analysis, over and above that contributed by predictors entered earlier in the analysis. Multiple regression analyses were deemed applicable here for three reasons. First, because interaction effects were tested, an ANOVA is likely to cause an unequal number of participants in each group. Second, a design with continuous variables makes it possible to use all available information (Cohen \& Cohen, 1985). Third, using multiple regression analyses is one way to overcome the inherent problems one is about to confront when determining appropriate cut-off scores for the classification of groups selected for comparisons (see Introduction). Accordingly, the combination of defensiveness and trait anxiety dimensions permits an approximation of any conclusion that can be drawn with reference to, for example, the general definition of repressors, or each one of the other groups 
according to the classification scheme of Weinberger et al. (1979). All continuous predictor variables were centered and their product was used to carry interaction information. Apart from Study I, when significant interactions were found, they were further explored by testing the simple slopes for significance as recommended by Aiken and West (1991). Regression lines were examined at one SD above and one SD below the mean of the other predictor(s). Multivariate outliers were defined as those having standardized residuals $\geq Z= \pm 2.5$ and were excluded from the analyses (StatSoft, 1994).

Table 1

Overview of demographics, focal predictors and outcome measures in the studies.

\begin{tabular}{|c|c|c|c|c|}
\hline $\begin{array}{l}\text { Demographics, predictors and } \\
\text { outcome measures }\end{array}$ & $\begin{array}{l}\text { Study I } \\
(n=167)\end{array}$ & $\begin{array}{l}\text { Study II } \\
(n=79)\end{array}$ & $\begin{array}{l}\text { Study III } \\
(n=79)\end{array}$ & $\begin{array}{l}\text { Study IV } \\
(n=42)\end{array}$ \\
\hline \multicolumn{5}{|l|}{ Demographics } \\
\hline Mean age (and SD) & $35.9(7.3)$ & $27.6(6.6)$ & $28.2(8.6)$ & $24.4(5.2)$ \\
\hline Gender (\% female) & 55 & 39 & 100 & 86 \\
\hline \multicolumn{5}{|l|}{ Predictors } \\
\hline Trait anxiety & $\mathrm{X}$ & $\mathrm{X}$ & $\mathrm{X}$ & $\mathrm{x}$ \\
\hline Social desirability & $\mathrm{X}$ & $\mathrm{X}$ & $\mathrm{x}$ & $\mathrm{X}$ \\
\hline Anxious state & & $\mathrm{X}$ & $\mathrm{x}$ & $\mathrm{X}$ \\
\hline SI masked ego threat & & & & $\mathrm{X}$ \\
\hline SI masked physical threat & & & & $\mathrm{X}$ \\
\hline SI masked ego positive & & & & $\mathrm{X}$ \\
\hline SI unmasked ego threat & & & & $\mathrm{X}$ \\
\hline SI unmasked physical & & & & $\mathrm{X}$ \\
\hline SI unmasked ego positive & & & & $X$ \\
\hline \multicolumn{5}{|l|}{ Outcome measures } \\
\hline SI masked social threat & & & $X$ & \\
\hline SI masked physical threat & & & $\mathrm{X}$ & $\mathrm{x}$ \\
\hline SI unmasked social threat & & & $X$ & \\
\hline SI unmasked physical threat & & & $\mathrm{X}$ & $\mathrm{x}$ \\
\hline SI masked illness-related & $\mathrm{X}$ & & & \\
\hline SI masked negative emotion & $\mathrm{X}$ & & & \\
\hline SI unmasked illness-related & $\mathrm{X}$ & & & \\
\hline SI unmasked negative emotion & $\mathrm{x}$ & & & \\
\hline SI masked ego threat & & & & $x$ \\
\hline SI masked ego positive & & & & $x$ \\
\hline SI unmasked ego threat & & & & $x$ \\
\hline SI unmasked ego positive & & & & $\mathrm{x}$ \\
\hline SAR masked threat pictures & & $\mathrm{X}$ & & \\
\hline SAR unmasked threat pictures & & $\mathrm{X}$ & & \\
\hline Emotional responses (SER) & & & & $\mathrm{X}$ \\
\hline Emotional responses (SC) & & & & $\mathrm{x}$ \\
\hline
\end{tabular}




\section{Summary of the studies}

\section{Study I}

Is defensiveness associated with cognitive bias away from emotional information?

\section{Purpose}

The relation between anxiety and cognitive bias for threat-related information has generated considerable interest during the last decade, and a lot of research clearly indicates that anxiety is associated with attentional bias toward threat-related information, both in clinical and non-clinical samples (e.g., Williams et al., 1997; Eysenck, 1997). An interesting question is if there are also personality variables that are associated with cognitive bias away from threatening information. Eysenck's theory (1997) specifically proposes that so-called "repressors" (i.e., people who score low on trait anxiety and high on social desirability - the latter being interpreted as defensiveness) have a cognitive bias opposed to the processing of threat. If this is true, one should expect the repressors to selectively attend away from threat-related words relative to neutral words. Although this effect has been observed in one study (Mogg et al., 2000a), previous findings with regard to these individuals have, however, been far from conclusive (Brosschot et al., 1999; Fox, 1994; Myers \& McKenna, 1996). Some limitations of these studies are that (a) they have only used unmasked words as part of the emotional Stroop task, and (b) they have only used student samples. The present study differs from these earlier ones both by (a) including masked words as part of the emotional Stroop task, and (b) using a community sample of randomly selected individuals in the age from 25 to 50 years. Furthermore, implicit memory was investigated as an additional measure of cognitive bias. Gender was also included in the analyses because gender-related effects on the emotional Stroop task have previously been reported in at least two studies (Stewart, Conrod, Gignac, \& Pihl, 1998; Wikström, Lundh, \& Westerlund, 2003).

\section{Method}

Selected from a community sample, 167 individuals (75 men and 92 women, mean age 35.9 years, $S D=7.3$ ) performed a combined emotional Stroop and tachistoscopic identification task with masked and unmasked words, which consisted of illnessrelated words, (pain, cancer, tumour), negative emotion (e.g., ludicrous, mourns, rage), and neutral control words (e.g., cable, domain, buyer). Self-report inventories incorporated in the study were trait anxiety and social desirability (KSP; Schalling, 1985). 


\section{Main results}

Age showed significant correlations with both colour naming speed $(r=.22, p=.003)$ and word identification $(r=-.34, p<.0001)$, and comparison between the genders revealed that the men identified more words than the women on the tachistoscopic identification task (48.4 vs. 43.6, $t[178]=2.24, p=.027)$, although there was no significant gender difference on colour-naming speed. Because of these effects of age, not only gender but also age was included in all subsequent analyses. To examine main effects and interaction effects of trait anxiety and social desirability (i.e., defensiveness), hierarchical multiple regression analyses were conducted on (a) colour naming speed and interference indices on the Stroop task, and (b) word identification performance, priming effects, and implicit memory bias indices on the tachistoscopic identification task. Age and gender were entered in step one. In step two, trait anxiety and social desirability were entered as predictors. In step three the interaction terms (cross products) of trait anxiety and social desirability, gender and trait anxiety, and gender and social desirability (standardised scores) were entered, and in step four the interaction term of gender, trait anxiety and social desirability was entered.

The Stroop task. On colour naming speed for unmasked words there was a significant increment in explained variance from step 2 to step $3, \Delta R^{2}=.05, F(3,157)=2.98, p=$ .033 , partially due to marginally significant interaction effect between gender and social desirability $(p=.066)$. To test if defensiveness correlates differently with word identification and colour naming, the differences between these correlations were analysed. Social desirability and colour naming of unmasked words correlated $r=.08$ $(p=.40)$ among the women, and $r=-.26(p=.028)$ among the men, which was a statistically significant difference, $z=-2.21, p=.03$. For masked illness words, there was a significant increment in explained variance from step 1 to step $2, \Delta R^{2}=.065$, $F(2,122)=4.28, p=.016$, due to a negative association between interference for masked illness words and social desirability $(\beta=-.22, p=.019)$. That is, the higher the participants' social desirability scores, the faster they were to colour name masked illness words relative to masked neutral words.

Implicit memory task. When overall word identification was used as the criterion, there was a significant increment in explained variance from step 2 to step $3, \Delta R^{2}=.04$, $F(3,158)=2.74, p<.045$, due to an interaction between gender and social desirability $(\beta=.20, p=.033)$. Analysing separately by gender, social desirability and word identification correlated $r=-.32(p=.002)$ among the women and $r=.06(p=.62)$ among the men, which again was a significant difference, $z=2.48, p=.01$.That is, women who scored higher on social desirability identified fewer words on this task.

\section{Discussion}

The most robust findings in the present study refer to the gender-specific associations between defensiveness and general performance on the emotional 
Stroop and tachistoscopic identification tasks. Thus, higher defensiveness (i.e., social desirability) among the men was associated with faster colour naming of unmasked words (independent of word category), whereas no such effect was found among the women. On the other hand, higher defensiveness among the women was associated with poorer identification of words in general (independent of word category and priming) on the tachistoscopic identification task - an effect that was not significant among the men. These gender-specific effects could perhaps be due to the presence of opposite-sex experimenter- effects that have been observed in previous research (Kline, Blackhart \& Joiner, 2002). Further, it is interesting to note that all effects of defensiveness on the Stroop task were in the direction of either a faster colour naming of words in general, or a faster colour naming of masked threat words as compared with neutral words (i.e., an emotional Stroop facilitation effect). This is consistent with the assumption that defensive information processing is associated with an attention away from emotional information, although this assumption may need to be broadened to an attention away from potentially threatening information (i.e., information which may be suspected of containing emotionally threatening meanings). One speculation is that the use of a community sample in the present study may have been responsible for part of this effect. Most earlier studies of repressive coping style and cognitive bias have used student samples, participants who are more familiar with the kinds of research activities that go on in psychology departments, including laboratory tests. It is unlikely that people from a community sample of the kind that was used in the present study have prior knowledge of this kind of experimental research. One possibility is that this unfamiliarity with the laboratory situation makes the testing situation itself into an emotional event. Although some weak content-specific effects were found on masked words, it may therefore be speculated that such participants may respond to the emotional testing context in a way that may override potential cognitive biases to the emotional contents of the separate words within the tests.

\section{Study II}

Unconscious responses to threatening pictures: The interactive effect of trait anxiety and social desirability on skin conductance responses.

\section{Purpose}

Enhanced skin conductance responses (SCRs) to fear relevant pictorial stimuli (relative to neutral) presented outside conscious awareness (i.e., masked stimuli) have been found to be a consistent effect in phobics (e.g., Öhman \& Mineka, 2001). Furthermore, the role of unconscious perceptual influence on autonomic responses has an apparent relationship to research on selective attention. In this paradigm, research data convincingly show that anxiety-prone individuals show selective attention to threatening information unavailable for conscious perception, which is further assumed to be a vulnerability factor that predisposes to anxiety disorders 
(e.g., Eysenck, 1997; Williams et al., 1997). Because biased processing of potentially threatening external cues play a central role in the definition of trait anxiety, it could possibly be one critical factor that determines the SCRs to masked threatening pictures. Even though there is, as yet, no direct evidence of a relationship between autonomic responses and selective attention to threatening stimuli, the main purpose of the present study was to explore whether trait anxiety also exerts influence on autonomic responses due to perception of pictorial threat. Additionally, social desirability (i.e., defensiveness) was also included in the analyses, as social desirability, according to Weinberger et al. (1979), is appropriate for splitting low and high trait anxiety groups into four groups. Thus, the present study was designed to investigate the role of trait anxiety and social desirability with regard to SCRs following presentation of masked and unmasked pictorial threat-related information in a non-clinical sample. That is, (1) in accordance with a previous finding (Najström \& Högman, 2003), we expected a main effect of stimulus type for subliminally presented pictures (higher SCRs for threat relative to neutral), and (2) we further explored if this main effect could be explained by the presence of high trait-anxious individuals in the sample.

\section{Method}

Selected from a student sample, 79 individuals (48 men and 31 women, mean age 27.6 years, $S D=6.6$ ) were presented with coloured pictures, which consisted of masked and unmasked, threat-related (e.g., mutilated bodies, battered women) and neutral control pictures (e.g., mushrooms on a field, umbrella). During the picture presentation, the participants' SCRs were recorded. Trait anxiety (STAI-T; Spielberger et al., 1983) and social desirability (16PF IM scale; Cattel et al., 1993) data were collected before each participant was scheduled for their first laboratory session. Prior to picture presentation, the participants' baseline skin conductance levels (SCL) were established. To target selective autonomic responses, threat indices were calculated for each individual by subtracting the mean range value (defined as the distance between the lowest and the highest skin conductance level during the measurement period) for neutral pictures (unmasked, masked) from the mean range value for threat pictures. To examine the main effects of trait anxiety and social desirability, and their interaction, hierarchical multiple regression analyses were conducted on the SCR threat indices (see below) separately for each exposure condition. Controlling for basic individual variation in SCL, baseline SCL (squareroot transformed) was entered in step one. Trait anxiety and social desirability were entered in step two, and the interaction term (cross product) of trait anxiety and social desirability (mean centered scores) was entered in step three. 


\section{Main results}

In accordance with our expectations, there was a main effect of stimulus type, with stronger SCRs in response to masked (i.e., subliminal) threatening pictures relative to neutral ones $(t=5.77, p<.0001)$. In addition, trait anxiety and defensiveness (as measured with social desirability) accounted for a large amount of explained variance to this effect $\left(\Delta R^{2}=.39, p<.0001\right)$. Specifically, whereas higher anxiety scores were associated with increased SCRs for threatening pictures (compared with neutral), higher scores on social desirability were associated with decreased SCRs for threatening pictures. Also, when the interaction term between trait anxiety and social desirability was entered $\left(\Delta R^{2}=.04, p=.048\right)$, it became clear that the main effect of trait anxiety was largely due to those participants who were simultaneously scoring low on social desirability $(p<.0001$ and $p=.012$, for the low and high, respectively). With regard to the unmasked pictures, as expected, there was a main effect of stimulus type, again with stronger SCRs in response to unmasked (i.e., supraliminal) threatening pictures relative to neutral ones $(t=5.12, p<.0001)$. In this exposure condition, was a significant increment in explained variance from step 1 to step 2, $\Delta$ $R^{2}=.10, F(2,72)=4.20, p=.019$, due to a positive association between threat index and social desirability $(\beta=.26, p=.051)$ and a positive association between threat index and trait anxiety $(\beta=.36, p=.006)$. That is, higher scores of both anxiety and social desirability, independently of each other, were associated with increased SCRs to threatening pictures (compared with neutral).

\section{Discussion}

The strong effect of trait anxiety (in combination with low social desirability) on SCRs to masked threatening pictures provides additional evidence to support the notion that preattentive processing of threat may be a vulnerability factor in trait anxiety that predisposes to anxiety disorders. Interestingly, despite being moderately high trait-anxious, elevated levels of social desirability virtually eliminated the effect of trait anxiety on SCRs seen among those scoring low on social desirability. This finding seems to lead to the conclusion that the importance of social desirability is quite crucial for those scoring higher on trait anxiety, and it is of theoretical importance to explain why these individuals are in fact having elevated trait anxiety levels despite the fact that their preattentive processing of threat suggests the opposite. Even though the main effects of stimulus type were approximately equal in effect size, trait anxiety and social desirability provided markedly less explained variance in the supraliminal exposure condition than in the subliminal one. Thus, it seems that effects are less dependent on trait variables under conditions where the pictures can be consciously perceived. The present study suggests that one has to detect elevated levels of anxiety in the sample in order to achieve increased SCRs to masked threatening stimuli (relative to neutral ones). Furthermore, even though conditioned responses are being more readily acquired by means of biologically relevant stimuli (Öhman \& Soares, 1993), it could be the case that the effects of conditioning are dependent on levels of anxiety. 


\section{Study III}

The interactive role of worried mood and trait anxiety in the selective processing of subliminally presented threat words.

\section{Purpose}

The emotional Stroop task has more or less become a household name in modern literature concerned with cognition and emotion. Evidence of Stroop interference (i.e., prolonged colour naming) for threat words as compared to neutral has been reported in anxiety-prone individuals (e.g., Eysenck, 1997; Williams et al., 1997 for reviews). Even though the focus in previous research with the emotional Stroop task has been on this interference effect, some participants are in fact faster to name the colour of threat word than neutral control words, and thereby showing an opposite facilitation effect in their processing of threat words (e.g., Mogg et al., 2000a; Lundh et al., 2001). Two possible hypotheses about this kind of effect can be found in the literature: (1) a state-trait interaction hypothesis, and (2) a hypothesis that repressive coping causes reverse cognitive biases. The state-trait interaction hypothesis was originally proposed by Williams, Watts, MacLeod and Mathews (1988). According to their theory, low trait-anxious individuals tend to divert their attention away from threat and this pattern is further strengthened by stress, whereas the opposite is the case for those high in trait anxiety. In accordance with this theory, MacLeod and Rutherford (1992) reported that elevated levels of stress among low trait-anxious individuals were associated with Stroop facilitation for masked threat words, whereas elevated levels of stress were associated with a Stroop interference effect for those high in trait anxiety. The repressive coping hypothesis has been clearly elaborated by Eysenck (1997), who specifically proposed that so-called "repressors" (i.e., people who score low on trait anxiety and high on social desirability - the latter being interpreted as defensiveness) have a cognitive bias opposed to the processing of threat, and may therefore be expected to show emotional Stroop facilitation. Curiously, no study has so far combined the state-trait hypothesis and the repressive coping hypothesis. The purpose of the present study was to therefore to study possible interactions between trait anxiety, defensiveness (social desirability), and level of stress on the emotional Stroop task. Level of stress in the present study was operationalised in terms of self-reported worried mood immediately before Stroop testing. The main purpose was to test the hypotheses that (1) elevated levels of stress (worried mood) are associated with colour naming facilitation of subliminally presented threat words in low trait-anxious individuals, in accordance with previous findings (MacLeod \& Rutherford, 1992), and (2) that this effect is due primarily to socalled repressors, that is high-defensive low trait-anxious participants. A second aim of the study was to investigate the test-retest stability of the emotional Stroop task. Thus, the temporal stability of biases in the subliminal condition was of particular interest because it has yet not been explored. Accordingly, each participant was scheduled to perform the Stroop task twice. 


\section{Method}

In a sample consisting of 79 female students, the participants performed the emotional Stroop task twice (within approximately four weeks), which consisted of masked and unmasked presentation of social threat words (e.g., ridiculous, stupid, inadequate), physical threat words (e.g., cancer, lethal, infected), and neutral words (e.g., domain, letter, number). Trait anxiety (STAI-T; Spielberger et al., 1983) and social desirability (KSP-SD; Schalling, 1985) data were collected before each participant was scheduled for their first laboratory session. Prior to the Stroop task, the participants reported their levels of worried mood on a visual analogue scale. Of these 79 individuals, two individuals were excluded due to technical problems with the apparatus during their first session in the laboratory. Two participants participated only in the first session. Thus, both the first session (mean age 28,0 years, $S D=8.4$ ) and the second session (mean age 28,4 years, $S D=8.6$ ) comprised 77 individuals. To examine main effects and interaction effects of worried mood, trait anxiety and social desirability, hierarchical multiple regression analyses were conducted on Stroop interference indices. Worried mood, trait anxiety and social desirability were entered in step one. In step two the interaction terms (cross products of mean-centered scores) of trait anxiety and social desirability, worried mood and trait anxiety, and worried mood and social desirability were entered, and in step three the interaction term of worried mood, trait anxiety and social desirability was entered.

\section{Main results}

At session 1, the multiple regressions showed significant effects only on the Stroop interference index for masked social threat words. For these threat words, there was a significant increment in explained variance from step 1 to step $2, \Delta R^{2}=.12, F(3,70)$ $=3.19, p=.029$, due to a marginally significant interaction term of worried mood and trait anxiety $(\beta=.23, p=.054)$. Simple slope analyses revealed that high trait-anxious participants demonstrated the expected increase in Stroop interference for masked social threat words due to elevated levels of worried mood $(t=1.92, p=.059)$, the likewise expected pattern of a decrement in interference due to elevated levels of worried mood for low trait-anxious was confirmed, although not significantly so $(t=$ $-1.23, p=.22)$. In absence of any significant interaction involving social desirability, this decrease in interference due to elevated worried mood among low trait-anxious individuals could not be explained by the presence of high-defensive individuals in this group (i.e., the repressors).

At session 2, the response patterns were reproduced for masked physical threat words. For these threat words, there was a significant increment in variance from step 1 to step $2, \Delta R^{2}=.12, F(1,37)=5.48, p=.025$, due to an interaction between worried mood and trait anxiety $(\beta=.37)$. Simple slope analyses demonstrated that, at lower levels of trait anxiety, worried mood was negatively associated with Stroop 
interference for masked physical threat words $(t=-2.02, p=.050)$, whereas at higher levels of trait anxiety, worried mood was positively associated with this interference index, although not significantly so $(t=1.62, p=.11)$. Further, although the interaction between worried mood and trait anxiety for social threat words failed to reach significance, the direction of effect among participants at lower levels of trait anxiety was the same as for physical threat words, with worried mood being negatively associated with Stroop interference for masked social threat words $(t=-$ $2.70, p=.010)$. In contrast to session 1 , the direction of the effect was not influenced by levels of worried mood among those at higher levels of trait anxiety $(p=.76)$. However, at session 2, all analyses involving trait anxiety and worried mood were carried out among low-defensive participants only $(N=41)$ because of very small variance in worried mood in the high-defensive group. The test-retest reliabilities for the Stroop indices were very low (unmasked social words, $r=.22$; unmasked physical words, $r=-.04$; masked social words, $r=.05$; masked physical words, $r=$ .10). Analyses split by group (low and high groups in defensiveness and trait anxiety) did not further clarify the picture for any of the indices.

\section{Discussion}

Although the interactions between worried mood and trait anxiety for masked threat words mostly went in the predicted directions, the effects were rather weak and only statistically significant in some cases. However, high trait-anxious individuals seemed to show a positive feedback loop, attending towards threat stimuli as a function of level of worried mood, with the result that their anxiety will tend to escalate in a dysfunctional manner, whereas the low trait-anxious individuals seemed to show a negative feedback loop, attending away from threat stimuli as a function of level of worried mood, with the result that their anxiety is kept at low levels. Also, the significant reduction in levels of worried mood from session 1 to session 2 for those at high levels of defensiveness in the present study demands explanation. The high-defensive participants seemed to approach session 2 with another strategy than the other groups. One possible speculation is that they to some degree "shut off" emotionally from the potentially anxiety-arousing test situation at session 2. Besides the obvious fact that our defensive participants were aware of the stimulus content involved, the group was also presumably alerted to the fact that their personal disposition was somehow involved. This might suggest the possibility that the emotional Stroop task may not measure the same processes at these two separate occasions. Whereas the experimental setting at the first testing session may be assumed to constitute a novel situation for most of the participants, this is definitely not the case at the second testing occasion, even if the external situation and the procedure are strictly identical. In fact, it could be argued that the first session measures cognitive biases in a "novel context" situation, whereas the second session measures cognitive biases in a "familiar context" situation. Additionally, the lack of test-retest stability of the emotional Stroop task, as seen in the present study, may either be due to contextual factors and different expectancies (e.g., a novel 
context vs. a familiar context), and/or to differing emotional states. In any case, however, this lack of stability suggests that Stroop interference should not be treated as a trait variable; cognitive bias for threatening stimuli as seen on the emotional Stroop task is probably the result of a complex combination of trait variables, temporary cognitive processes and emotional states.

\section{Study IV}

Information-processing and emotional responses to stress: An investigation using self-report and physiological measures of stress.

\section{Purpose}

Selective attention to threatening information has received increased attention because recent cognitive formulations have placed much emphasis on its role as a key cognitive factor underlying vulnerability to and the maintenance of anxiety disorders (e.g., Eysenck, 1997). Others have suggested that vulnerability to anxiety is concerned with biological factors (e.g., McNaughton \& Gray, 2000), or that various trait factors, such as, negative affectivity (e.g., Clark et al., 1994) may play a key role in the development of clinical anxiety. It is notable that selective attention and trait factors should not be treated as divorced from each other, as selective attention to threat cues are typically seen in high-anxious individuals. Our preference in the current study was once again to focus on the response patterns for subliminally presented stimuli. The relationship between selective attention to threatening material (subliminally presented) and subjective emotional responses to stressors seems to be established. However, its possible relationship to the physiological responses to stressors is yet to be clarified. The main purpose of the present study was to investigate the relative contributions of trait anxiety and Stroop interference for masked threat words (i.e., preattentive bias) to emotional responses (subjective emotional responses, and autonomic reactivity) following exposure to a laboratory stressor (a problem-solving task, to be performed under time pressure).

\section{Method}

The sample consisted of 42 students (36 women, mean age 24.4 years, $S D=5.2$ ). The participants performed the emotional Stroop task, which consisted of masked (subliminal exposure) and unmasked (supraliminal exposure) presentation of ego threat words (e.g., stupid, worthless), physical threat words (e.g., cancer, lethal), ego positive words (e.g., gifted, smart), and neutral words (e.g., domain, letter, number). Trait anxiety (STAI-T; Spielberger et al., 1983) and social desirability (KSP-SD; Schalling, 1985) data were collected before each participant was scheduled for their first laboratory session. After to the Stroop task, in another laboratory, the participants performed a stress task where the participants' skin conductance activity was recorded. Electrodermal measures included were skin conductance variance 
(SCV) and skin conductance level (SCL). The SCV variable was defined as the variance of SC activity over the measurement period, whereas the SCL variable was defined as the mean tonic SC activity. The electrodermal activity defining each of these two variables was continuously assessed during the task. Immediately after the stress task, the participants reported their subjective emotional experience on six visual analogue scales (SER: subjective emotional responses). To examine reactivity to the stressor, residual scores (the SER was regressed on pre-task state anxiety, and task skin conductance values were regressed on their baseline values) were used to index each individual's changes in anxious state and skin conductance activity. For each exposure condition, three Stroop interference indices were calculated for each individual by subtracting the mean colour naming latency for neutral words (unmasked, masked) from the mean colour naming latency for emotional words (ego threat: unmasked, masked; physical: unmasked, masked: ego positive). To examine the main effects of trait anxiety and Stroop interference, and the interaction between trait anxiety and Stroop interference, hierarchical multiple regression analyses were conducted on subjective emotional responses (SER), and skin conductance reactivity data (SCV and SCL). Trait anxiety was entered in step one. In step two, masked Stroop interference index was entered. In step three, unmasked Stroop interference index was entered. Finally, in step four, the interaction terms (cross product) of trait anxiety and Stroop interference index (centered scores) for both masked and unmasked words were entered. This procedure was performed for each emotional word category - Stroop interference indices (masked, unmasked) for ego threat, physical threat, and ego positive words.

\section{Main results}

When the SER (subjective emotional responses) variable was used as criterion, and when Stroop interference indices for ego threat words were included as predictors, there were no significant effects of trait anxiety, but a significant increment in explained variance due to the effect of Stroop interference for masked ego threat words $\left(\Delta R^{2}=.19, p=.003\right)$. Similarly, when Stroop interference indices for physical threat words were included as predictors, there was a significant increment in explained variance due to the effect of Stroop interference for masked physical threat words $\left(\Delta R^{2}=.22, p=.002\right)$. Descriptively, higher interference scores were associated with higher levels of self-reported emotional distress (as measured by the SER) in response to the stressor. Further, there was no significant increment in explained variance due to the inclusion of Stroop interference for unmasked threat words, or to the inclusion of the two interaction terms of trait anxiety and Stroop interference for threat words (masked, unmasked).

When the SCV (skin conductance variance) variable was used as criterion, and when Stroop interference indices for ego threat words were included as predictors, there were no significant effects of trait anxiety, but a significant increment in explained variance due to the effect of Stroop interference for masked ego threat words $\left(\Delta R^{2}=\right.$ $.16, p=.009)$. Similarly, when Stroop interference indices for physical threat words 
were included as predictors, there was a significant increment in explained variance due to the effect of Stroop interference for masked physical threat words $\left(\Delta R^{2}=.15\right.$, $p=.010)$. Descriptively, higher interference scores this time being associated with lower autonomic reactivity (as measured by the SCV) in response to the stressor. Again, there was no significant increment in explained variance due to the inclusion of Stroop interference for unmasked threat words, or to the inclusion of the two interaction terms of trait anxiety and Stroop interference for threat words (masked, unmasked). In addition, Stroop interference for masked physical threat words was also significantly negatively associated with SCL $(p=.010)$, but the negative association between Stroop interference for masked physical threat words and SCL failed to reach significance $(p=.20)$. Stroop interference indices for ego positive words were not significantly associated with any of the emotional response outcome measures (i.e., SER, SCV, and SCL).

When the associations between Stroop interference and questionnaire scores prior to the stressor were analysed, the multiple regressions showed significant effects only on the Stroop interference index for ego threat words. For masked ego threat words, there were no significant effects of trait anxiety and social desirability, but a significant increment in explained variance due to a highly significant interaction term of trait anxiety and social desirability $\left(\Delta R^{2}=.19, p=.004\right)$. The significant interaction was further explored by testing the simple slopes for significance, using trait anxiety as the focal predictor. Regression lines were examined at one SD above and one SD below the mean of social desirability. These analyses showed that higher scores on trait anxiety were strongly associated with an increase in Stroop interference for masked ego threat words (compared with neutral) among those at lower levels of social desirability $(t=3.21, p=.003)$, and negatively associated among those at higher levels $(t=-1.66, p=.10)$. Furthermore, although the interaction effects failed to reach significance for masked ego positive and physical threat words (both indices, $p=.17$, respectively), the direction of effects was the same as for masked ego threat words as described above.

\section{Discussion}

The present results replicate the previous finding (e.g., Pury, 2002) that Stroop interference for subliminally presented (i.e., preattentive bias) threat words significantly predicts the subjective emotional response to a laboratory stressor. That is, higher scores on preattentive bias were consistently (across types of threat) associated with an increase in emotional distress, and at a reasonably high magnitude of effect. In contrast, when the subjective measure of emotional response to stress was exchanged with the objective one (i.e., skin conductance activation), the effect of preattentive bias was diametrically opposite - Stroop interference for masked threat words (again across types of threat) this time being negatively associated with autonomic responding to the very same stressor. Also, neither of these effects were a function of pre-existing anxiety levels. With regard to Stroop interference for masked words, individuals at higher levels of trait anxiety (coupled 
with low scores on social desirability) were highly overrepresented among those at higher levels of preattentive bias for threat words.

The finding of reduced autonomic responding for individuals with elevated levels of preattentive bias is remarkably similar to such findings showing that the anxiety patients' changes in skin conductance responses are smaller than those of the nonanxious controls when exposed to moderately stressful events (Hoehn-Saric \& McLeod, 2000, for a review). Furthermore, similar effects were found in high traitanxious individuals following exposure to stressful events (e.g., Wilken et al, 2000). Considering the autonomic inflexibility, not only seen in anxiety patients (HoehnSaric \& McLeod, 2000, for a review), but also in worry (Thayer, Friedman, \& Borkovec, 1996), the inability to effortlessly inhibit negatively valenced verbal thought activity seen in worry could perhaps correspond to the effect of preattentive bias (i.e., the inability to automatically inhibit the processing of threatening language cues) that, in addition, may well be the vulnerability marker in worry and anxiety. In other words, both worrying and preattentive processes may continuously activate cognitive anxiety networks. At or above a certain level of activation, these networks lead to a positive feedback loop in which the perception of anxiety cues leads to increasingly more perception of anxiety. Of course, the autonomic data in the present study were exploratory and should be interpreted accordingly. Needless to say, the present autonomic findings need to be replicated, and the skin conductance data should preferably be complemented with additional physiological measures. 


\section{General discussion}

One question raised in the Introduction was whether it is possible to identify some factors that could assist us in determining whether an individual will show preferential preattentive processing towards or away from threat-related information. A specific combination of trait anxiety and social desirability was rather consistently associated with effects in the same direction, and social desirability (i.e., defensiveness) on its own was found to produce some consistent trends across different studies. Another question raised in the Introduction was whether it is possible for preattentive bias could to be used as a predictor of physiological responses during stressful events. On the basis of the present results, it appears that this would be the case. The results will be discussed in greater detail below, and some possible explanations for some inconsistencies across different studies will be offered.

\section{Predicting preattentive processing of threatening information}

If we start by considering the overall pattern for those at higher levels of trait anxiety, it is clearly the case that higher trait anxiety scores are associated with increased preattentive processing of threatening information. Specifically, apart from Study I, the studies rather consistently provided evidence supportive of this claim. To briefly recapitulate, in Study I, trait anxiety did not make any significant contribution to Stroop interference. In Study II, elevated levels of trait anxiety were associated with an increase in SCRs to masked threatening pictures (relative to neutral ones). This latter effect, however, was seen mainly among participants who simultaneously scored low on social desirability. In Study III at session 1, while high trait-anxious participants demonstrated the expected increase in Stroop interference for masked social threat words due to elevated levels of worried mood, the likewise expected pattern of a decrement in interference due to elevated levels of worried mood for low trait-anxious participants was confirmed. Furthermore, there was no evidence that these effects were modified by social desirability (i.e., defensiveness). Although this pattern was not reproduced for masked physical threat words, unreported data showed that, in competition with the Trait Anxiety X Worried Mood variable, the interaction between trait anxiety and social desirability (defensiveness) shows a tendency towards significance $(p=.11)$. When correlational analyses were performed separately within the low and high trait anxiety groups, there was a negative association between Stroop interference for masked physical threat words and social desirability $(r=-.48)$ in the high trait-anxious group (STAI-T $\geq 45)$. Descriptively, the "true" high trait-anxious showed Stroop interference, whereas the defensive high trait-anxious showed facilitation. Furthermore, at session 2, the interactive effect of trait anxiety and worried mood was on the whole reproduced among low-defensive individuals. In Study IV, higher scores on trait anxiety were strongly associated with an increase in Stroop interference for masked ego threat words (compared with neutral) among those at lower levels of social desirability and were negatively 
associated among those at higher levels. Descriptively, whereas the "true" high traitanxious group showed a substantial Stroop interference effect, the "true" low traitanxious group showed a substantial Stroop facilitation effect. Furthermore, although the interaction effects failed to research significance for masked ego positive and physical threat words, the direction of effects was the same as for masked ego threat words, as described above.

Although the topic under this heading is concerned with content-specific effects on masked words, a consideration of the overall pattern for those at higher levels of social desirability, in most cases the effects point in the same direction - attention away from emotional information. With regard to content-specific effects, in Study I, higher levels of social desirability were associated with a decrease in Stroop interference for masked illness-related words. In Study II, higher levels of social desirability were strongly associated with lower SCRs to masked threat pictures (relative to neutral ones). In Study III, there were no straightforward effects of social desirability, but as noted above, there was a negative association between Stroop interference for masked physical threat words and social desirability in the high traitanxious group only. In Study IV, there was the strong interactive effect of trait anxiety and social desirability on masked ego threat words. Specifically, there was a strong negative association between Stroop interference and social desirability among participants at higher levels of trait anxiety - an effect consistent with the effect for masked physical threat words in Study III. Furthermore, another interesting finding was the negative association between Stroop interference and trait anxiety among participants at higher levels of social desirability. This means that the defensive high trait-anxious showed less Stroop interference compared to the repressor group. Descriptively, whereas the repressor group's bias scores were close to zero, the former group showed a substantial Stroop interference effect that, in addition, was as large as that seen in the "true" low trait-anxious group. The direction of effects was the same for ego positive and physical threat words, but not significantly so.

It is interesting to note that all effects of defensiveness on the Stroop task were in the direction of either a faster colour naming of words in general, or a faster colour naming of threat words as compared to neutral words (i.e., an emotional Stroop facilitation effect). This is consistent with the assumption that defensive information processing is associated with an attention away from emotional information. However, considering that the male participants were generally faster to colour name words (irrespectively of valence) in Study I, this assumption may need to be broadened to an attention away from potentially threatening information (i.e., information which may be assumed to containing emotionally threatening meanings). One possible explanation is that the participants in Study I underwent a structured interview prior to the Stroop task, and were presumably alerted to the fact that their personal disposition was somehow involved. This, in turn, could perhaps be viewed as a context that poses a threat to their self-concept. At the same time, in Study III, participants with high levels of defensiveness seemed to approach session 
2 with another strategy than those low in defensiveness did, as seen in their significant reduction in levels of worried mood from session 1 to session 2 . Consequently, this may indicate that expectancies play an important role in the activation of defensive processes in individuals who are high on trait defensiveness.

As noted in the Introduction, the role of unconscious perceptual influence on autonomic responses has an apparent relationship to research on selective attention. Because biased processing of potentially threatening external cues plays a central role in the definition of trait anxiety, we recognised the possibility that this variable exerts influence of an autonomic outcome measure as well. Indeed, the results showed that only individuals with elevated levels of trait anxiety (in combination with low levels of social desirability) showed a marked enhancement of SCRs to subliminally presented threatening pictures (compared to neutral ones). This further suggests the possibility that one must detect elevated levels of anxiety in the sample in order to achieve increased SCRs to different types of threatening stimuli in general. It is also notable that, despite previous claims (e.g., Öhman, 1996) that, when subliminal presentation is used, the arousal system should only be activated by biologically relevant naturalistic stimuli, two recent studies demonstrated that subliminally presented emotional words (which have been deemed to possess low threat value) elicited significantly stronger SCRs than did neutral words. Specifically, stronger SCRs were elicited by masked threat words (compared to neutral words) among spider phobics (Van den Hout, van Jong, \& Kindt, 2000), and by masked negatively valenced words in normal participants (Silvert, Delplanque, Bouwalerh, Verpoort, \& Sequire, 2004). Unfortunately, the latter study did not investigate whether the main effect of stimulus type could be explained by the possible influence of trait variables.

\section{Predicting emotional responses to a stressful event}

Given the number of studies that have investigated the relationship between preattentive bias and emotional distress in response to emotionally stressful events, it is somewhat surprising, however, that no attempt has yet been undertaken to find evidence of a relationship between preattentive bias and physiological responses. First, Study IV produced results consistent with other studies (e.g., Pury, 2002) preferential preattentive processing of threat cues is associated with an increased tendency to respond with emotional distress to a stressor. Second, in response to the same stressor, when the relationship between preattentive bias and a physiological indicator (i.e., autonomic reactivity) of emotional responses was examined, the direction of effects was reversed. Because the inclusion of a physiological outcome measure was a novel aspect, the latter effect demanded an explanation. Much of the interpretations for the finding that higher preattentive bias scores being associated with diminished autonomic reactivity were formed on the basis of similar findings from studies that have exposed clinically anxious individuals (or high trait-anxious individuals) to stressful events. In particular, anxiety patients' changes in SC activity have been found to be smaller than those of non-anxious controls when exposed to 
moderately stressful events (Hoehn-Saric \& McLeod, 2000, for a review). These authors (see also Friedman \& Thayer, 1998; Thayer et al., 1996, for a similar conclusion) argued that this "autonomic inflexibility" seen in anxiety patients reflects a less adaptive response to stress (i.e., ineffective emotional regulation, and behavioural inflexibility). Because this effect is not disorder-specific as it has been observed across different anxiety disorders, it was discussed in relation to preattentive bias, another "non-disorder-specific" effect that has been rather convincingly demonstrated among clinically anxious and high trait-anxious people. The effect of diminished autonomic reactivity due to preferential preattentive processing was discussed in terms of an inability to automatically inhibit the processing of threatening language cues that could correspond to the effects seen in anxiety and worry (i.e., failure to effortlessly inhibit negatively valenced verbal thought activity). It has been suggested (e.g., Borkovec, 1994) that the constant worry seen in patients with generalised anxiety disorder is triggered by cues (external and/or internal) that are associatively related to upcoming events (those that they fear). Thus, preattentive processing may continuously activate cognitive anxiety networks. At or above a certain level of activation, these networks lead to a positive feedback loop in which the perception of anxiety cues leads to increasingly more perception of anxiety. In addition, since these preattentive cues also activate physiological responses, these preattentively activated bodily responses have no obvious origin (i.e., the source of the responses fails to be consciously represented) and are thus an additional source that might trigger anxiety. Thus, it seems that preattentive bias predicts the prevalence of negatively valenced verbal thought activity that exists during a stressful event which will not only have an effect on the subjective emotional experience, but also on the physiological responses. Yet again, as earlier noted (Study IV), it should be remembered that the relationship between preattentive bias and the autonomic outcome measure was exploratory, and should therefore be interpreted accordingly.

\section{Is the preattentive bias for threat a vulnerability factor in the development of anxiety disorders?}

Despite the fact that preattentive bias is seemingly causally related to emotional distress in response to stressful events (Mathews \& MacLeod, 2002), whether this bias is ultimately sufficient for the development of clinical anxiety, and to what extent this bias translates specifically to one anxiety disorder over another remains to be clarified. Additionally, most theories that hold biased attentional processing as a factor responsible for mediating the vulnerability to anxiety disorders do not distinguish between types of anxiety and, hence, biased attentional processing is treated as a general vulnerability factor for anxiety.

According to Eysenck's (1997) theory, there is continuity between high trait-anxious people and clinically anxious patients in terms of cognitive biases and, hence, high trait-anxious individuals are more vulnerable to the development of generalised 
anxiety disorder. That is, high trait-anxious individuals have the tendency to process information in a threatening manner, especially when stressed, but a number of relevant stressors are also needed to trigger the onset of an anxiety disorder. When exposed to stressful life events, these individuals are more likely to selectively process the most threatening aspects of all the information available. Consequently, this biased processing style will result in disproportionately intense anxious responding to subsequent stressful life events. Also, generalised anxiety disorder patients exhibit preattentive a bias for negative information in general, rather than content-specific effects (Bradley, Mogg, White, \& Millar, 1995; Mogg et al, 1993). With the exception of generalised anxiety disorder, the critical issues here are why this processing style in some cases is restricted to specific categories of cues, and why people possess a processing style that favours negative information in the first place. As noted earlier, previous research shows that processing biases can be induced rather readily (MacLeod et al., 2002; Mathews \& Macleod, 2002). According to these authors, when people who preferentially process negative information are exposed to a stressful event, any negative thoughts that are cued should be preferentially attended. In this case, "attention to negative self-referent thoughts is likely to promote access to other associated autobiographical memories or images" (pp. 350). Furthermore, Rapee and co-workers (Hudson \& Rapee, 2000; Rapee \& Heimberg, 1997) suggested that genetic factors may account for people's general tendency to process information in a threatening manner, but environmental family factors could determine which specific cues receive increased attention. Of further relevance, a recent study (Richards \& Blanchette, 2004) found that the high trait-anxious group showed a Stroop interference effects for non-words (i.e., no pre-existing semantic associations) that have been negatively conditioned. Thus, whether this general emotional reactivity to stressful events will be manifested in any specific anxiety disorder is seemingly dependent on a number of factors, such as information available for processing during stressful events and an individual's specific learning history, but is also presumably dependent on other factors as well (e.g., biological vulnerabilities).

Although the topics in this section have to a large extent been restricted to effects of preattentive processing of threatening material, individual differences in effortful attentional control seems to be another factor underlying attentional processing. For example, Derryberry and Reed (2002) showed that high trait-anxious participants with poor attentional control experienced difficulty in disengaging from potentially threatening cues relative to those with good attentional control. In a recent paper, Mathews (2004) extensively discusses the interactive role bottom-up and top-down processes in anxiety-linked processing biases (see also, for example, Mackintosh \& Mathews, 2003; Mathews \& Macleod, 2002). According to this interaction hypothesis, in Mathews' words, "if the threat signal is strong enough, as in highly fearful or anxious individuals, the threat distracter is likely to capture attention...if top-down control is strong enough, then the representation of the threat distracter can be inhibited, and task performance continues without interruption" (pp. 1022). Thus, whether a stressful event would lead to the overrepresentation of threat cues within 
the information that anxious individuals encode from their environment is, according to this view, dependent on two effects - the strength of bottom-up signals associated with fear/anxiety, or the strength of top-down control, or both.

In conclusion, a person's most common emotional reaction when encountering a stressful event may be causally influenced by that person's habitual tendency to selectively encode the most threatening aspects of all the information available. Consequently, this biased processing style will result in disproportionately intense emotional reactions to subsequent stressful life events. However, prospective studies are required in order to determine whether this bias is ultimately sufficient for the development of clinical anxiety, and the extent to which this bias translates specifically to one anxiety disorder over another.

\section{The importance of trait anxiety and social desirability in preferential preattentive processing}

At this stage of the discussion it is important to remember that, apart from Study I, Studies II and III produced results that point to the fact that elevated levels of trait anxiety promote preferential preattentive processing of negatively valenced information, whereas elevated levels of social desirability generally prevent preferential preattentive processing of negatively valenced information, irrespective of outcome measure used. As a consequence, we would therefore expect these individuals to show more emotional distress when confronted with a stressful event. An analysis of the data in Study IV indeed revealed that this was the case. Specifically, the individuals at higher levels of trait anxiety (coupled with low scores on social desirability) were highly overrepresented among those at higher levels of preattentive bias. If, in fact, selective attention reinforces anxiety, selective attention towards potential threat cues in the environment is indeed dysfunctional in the sense that it may force some individuals into a vicious circle (e.g., Eysenck. 1997; Mathews, 2004). For instance, on the basis of findings from the studies in this thesis, we would expect high trait-anxious people to have an increased tendency (i.e., constituting a baseline condition) to process negatively valenced cues without being consciously aware of the information being processed. When confronted with a stressor, increased processing of negatively valenced cues (internal and/or external) will subsequently increase anxiety levels (due to the association between preattentive bias and emotional responses to a stressor). The increased anxiety levels are then likely to further reinforce selective attention, and the increased attention to negatively valenced cues will even further increase anxiety levels, and so on. Taken together, these effects logically support the conclusion that higher levels of trait anxiety (coupled with low scores on social desirability) do generally forecast emotional vulnerability.

Negative associations between social desirability and preferential preattentive processing among individuals at higher levels of trait anxiety have now been 
demonstrated on two independent outcome measures. What makes these findings particularly interesting is that they seem to lead to the conclusion that the importance of social desirability among high trait-anxious individuals is quite significant. The above conclusion could be further considered in at least two ways. First, social desirability should always be incorporated into the design when the focal interest of the study is effects of trait anxiety in order to obtain a "purer" measure of trait anxiety, but may also be useful in clinically anxious samples. Second, the effects of the "defensive high anxious" individuals are by definition interesting. As noted earlier, preferential preattentive processing of threat is a prominent feature in fear and anxiety that, in addition, has been suggested to be a factor in the development of clinical anxiety (e.g., Öhman \& Mineka, 2001, Williams et al., 1997). Therefore, it is of theoretical importance to explain why these individuals are in fact trait anxious despite the fact that their preattentive processing of threat suggests the opposite. Considering that evidence for preferential preattentive processing of threatening information in anxiety is rather compelling, one explanation could be that the factors that mediate the development of anxiety are not the same as for the "true" high traitanxious. A second explanation could be that the factors that mediate the maintenance of anxiety are not the same as for the "true" high trait-anxious. That is, once an individual with elevated levels of social desirability has become "trait anxious", activation of defensive processes in these individuals serves to avoid further processing of external threat cues.

Furthermore, although obviously speculative, it could be that high-defensive individuals' defensive behaviour is efficient in inhibiting the processing of negatively valenced information in situations in which these individuals anticipate an emotionally threatening event. Accordingly, it may be the case that effects of defensiveness are restricted to specific contexts. Baumeister and Cairns (1992), for example, tested repressors and non-repressors in a private and a public condition, and found effects only in the private condition. Participants were tested in pairs with trait anxiety/defensiveness scales administered by computer. After they had completed the scales the computer produced a bogus personality profile - either favourable or unfavourable - supposedly based on the test. In the private condition, participants were reassured that no one would ever see the profile and that the data would be used to investigate broad statistical trends. In the public condition participants were told that these data would be shown to their experimental partners. All participants were left alone to read their profile at their leisure. Repressors who received unfavourable feedback privately spent the least amount of time reading it, whereas repressors who received the same feedback publicly spent a long time reading it. They also spent less time reading the unfavourable compared to the favourable evaluation. This suggests that repressors used an avoidant strategy to dismiss negative information concerning the self in the private condition, but not in the public condition (see also, Derakshan \& Eysenck, 2005, for a similar finding). If this applies to defensive individuals in general, one could therefore speculate that such participants (i.e., defensive high anxious) may respond to the emotional testing context in a way that will selectively inhibit negatively valenced emotional information, or use other strategies that will serve to 
override any potential of being emotionally affected by the upcoming event. If this is correct, it suggests the possibility that the differential effects seen in the two high trait-anxious groups ("true" high anxious and defensive high anxious) on two different outcome measures in the present studies might only be restricted to contexts equivalent to testing contexts (i.e., anticipation of threatening events), and that these two high trait-anxious groups are thus equally likely to preferentially process environmental threat cues (occurring outside conscious awareness) in the case in which no such event is expected.

\section{Temporal stability of Stroop interference indices}

Stroop interference indices showed very little test-retest stability from the first to the second occasion of testing. Even if interference indices in the subliminal condition may be more reliable measures of biased attentional processing, the present data indicate that they are not more stable over time. In fact, very weak evidence was found that the participants (independent of disposition) have an attentional pattern that is stable over time; this was found in the supraliminal condition. The present data indicate that preattentive biases, as seen in Stroop interference effects of masked threat words, are influenced by temporary states of mood, and that these effects differ as a function of trait variables (see also, MacLeod \& Rutherford, 1992; Rutherford, MacLeod, \& Campbell, 2004). It is interesting to note that, although there was no temporal stability of Stroop effects for masked words at the individual level (i.e., when temporary state was not considered), the results from test occasion 1 to occasion 2 showed much more consistency when the interaction between trait and state variables were considered - with the exception of the high-defensive individuals. The results also suggest the possibility that the emotional Stroop task may not measure the same processes at these two separate occasions. Whereas the experimental setting at the first testing occasion may be assumed to constitute a novel situation for most of the participants, this is definitely not the case at the second testing occasion, even if the external situation and procedure are strictly identical. In fact, it could be argued that the first session measures cognitive biases in a "novel context" situation, whereas the second session measures cognitive biases in a "familiar context" situation. Further, the results suggest that this discrepancy between a novel context and a familiar one may be most important to participants with a repressive coping style, who show different patterns of responding at these two occasions.

To date, there are only a few studies that have investigated the test-retest reliability of the emotional Stroop task. Data suggest that bias for threat is seemingly stable in spider phobic individuals but not in controls (e.g., Kindt, Bierman, \& Brosschot, 1996; Kindt \& Brosschot, 1998). However, to the best of my knowledge, the temporal stability of subliminal indices has not previously been explored, and it is yet unclear whether this bias is stable over time in clinically anxious patients. It is interesting to note that, in Study III, this bias was unstable over time even in participants classified 
as being high trait-anxious. Of importance in the present context is that Stroop interferences scores for masked threat words have been employed in a small number of studies to assess treatment effects (Devineni, Blanchard, Hickling, \& Buckley, 2004; Mogg et al., 1996; Van den Hout et al., 1997). Apart from the Devineni et al. study (2004), this research indicates that biases in selective attention in anxious patients are significantly reduced after successful cognitive therapy. If the Stroop interference effects are dependent on context (i.e., novel vs. familiar), familiarisation with test procedures is a potential confound in the assessment of treatment effects. Taken together, assuming the impact of expectancies, at any test occasion, the influence of temporary states of mood and strategic behaviours may play a crucial role for the outcome. An alternative approach to assessing the stability of cognitive biases could be to retest the same individual in a slightly different context, by for example, switching the experimenter and/or performing the task in another setting. Thus, in line with the reasoning above, this procedure might create the means to assess the stability cognitive biases for novel context conditions. In the case of assessing treatment effects, it could be useful to obtain Stroop data on two separate occasions prior to treatment. An alternative approach would be to obtain both pre-treatment and post-treatment Stroop data in conditions in which biases for masked words are most likely to occur - when the participants are highly stressed, as processing biases are assumed to be elicited by stressful events (e.g., Mathews, 2004, Mathews \& MacLeod, 2002).

\section{Preferential preattentive processing: An extended discussion}

According to Mogg and Bradley (1998), if the threat value is sufficiently high, selective attention to the threat cues should be present in everyone, whereas relatively mild threat should attract attention only in high trait-anxious people. In order to account for this proposal, these authors argued that trait anxiety reflects the reactivity of a valence evaluation system to aversive stimuli. Put differently, selective attention to relatively weak threat cues is assumed to be a result of bias in the operation of the valence evaluation system in high trait-anxious people who, in turn, are assumed to be the primary factor responsible for mediating vulnerability to emotional disorders. If effects of selective attention occur as a consequence of something that could be conceptualised as evaluative bias, in the sense that these individuals evaluate threatening stimuli as more threatening than do those who do not possess evaluative bias, there should be (1) a quantitative difference between these sets of individuals in response to the presentation of threatening material (relative to non-threatening material), and (2) a quantitative difference between the intensities of different threat stimuli (i.e., threat value) in everyone. As noted in the Introduction, there are studies supporting the view that highly threatening cues produce selective attention effects that are significantly stronger than both neutral and mildly threatening pictorial stimuli (Koster et al., 2005; Mogg et al., 2000b; Wilson \& MacLeod, 2003) in all individuals. This does indeed suggest that the responses are related to the degree to which the stimulus content is being evaluated 
with respect to its threat values. As also noted earlier, many researchers have placed much emphasis on stimuli that constitute a biologically relevant threat (e.g., Mathews \& Macintosh, 1998; Mathews \& MacLeod, 2002; Mogg \& Bradley, 1998; Öhman \& Mineka, 2001). Yet, it is puzzling why (1) words, which are assumed to possess only mild threat value, produce Stroop interference effects that equal the magnitude of effects seen when naturalistic stimuli (i.e., pictures) corresponding to the participant's particular fear are presented (Kindt \& Brosschot, 1997), and (2) threat-related words, when masked to prevent conscious representations, elicit significantly stronger SCRs than do non-threatening words, even among normal participants (Silvert et al., 2004). What also remains to be explained is why there was no evidence of highly threatening pictorial stimuli (such as snakes and spiders) eliciting significantly stronger SCRs than non-threatening stimuli (flowers and mushrooms) among non-anxious individuals (Öhman \& Soares, 1994), as one would perhaps also expect that in all of us, snakes and spiders would have a higher threat value than would flowers and mushrooms. Accordingly, masked pictorial presentation of naturalistic stimuli is seemingly not sufficient to trigger differential SCRs unless anxiety levels are elevated (Study II). With reference to differential SCRs to masked words, even though this possibility was not tested, it could be the case that those at higher levels of trait anxiety were entirely responsible for the main effect of stimulus type in the study by Silvert and co-workers (2004). On the basis of this, it seems that variations in the threat value of the threat stimuli are not intimately linked to the responses seen on a cognitive outcome measure, or on an autonomic outcome measure.

It is clearly the case that evaluative mechanisms are involved in consideration of the evidence that some individuals under some conditions selectively attend to threatening information whereas others selectively attend away from threatening information, despite the fact that the stimulus content is of highly threatening nature. In addition, as noted earlier, the findings with regard to quantitative differences between the intensities of different threat stimuli are also supportive of the view that evaluative mechanisms are involved. Of importance in the present context, a recent study investigated the effects of stimulus intensity of unmasked pictorial threat cues in low and high trait anxiety (Koster et al., 2005). In the condition in which the set of pictures was deemed to possess the highest threat value, the magnitude of bias (favouring threatening pictures) in the low trait-anxious group was equivalent to that seen in the high trait-anxious group. Furthermore, in the condition in which the set of pictures was deemed to possess the highest threat value, on the basis of their normative valence and arousal rating, the pictures were, in fact, less arousing compared to those pictures used in Study II. Thus, despite assumed to possess high threat value, when these pictures were presented subliminally, they were not sufficient to cause a marked increase in SCRs among low trait-anxious individuals. In other words, the absence of an increment in SCRs for all participants in response to highly threatening pictures (e.g., mutilated bodies) compared to neutral pictures (e.g., mushrooms) is partially inconsistent with the evaluative bias hypothesis, as SCRs would be expected to change noticeably in all participants following such an 
increment in threat value of the stimulus content. In the present context, however, one should keep in mind that the investigations of the relationship between stimulus intensity and biased processing have only been on threat cues available for conscious awareness. That is to say, we do not know if the effects of stimulus intensity of the threat cues also hold in conditions in which these cues remain unavailable for conscious representations. On a more general note, when relating findings to the theoretical accounts given for the underlying mechanisms of selective attention, one should not fail to recognise the fact that one must distinguish between effects of different exposure conditions, and thereby not treat findings based on conscious and unconscious perception of the stimulus material as if the underlying dynamics were of the same kind. In fact, regardless of whether the outcome measures were based on latency data or autonomic data in the present thesis, the threat indices for masked and unmasked threat-related stimuli did not correlate, and therefore seem to measure different kinds of processes (see also, Lundh et al., 2001).

It seems misplaced to over-speculate about preattentive effects seen on the latency and autonomic outcome measures, as these studies provided too little data on which to base any conjecture. Nevertheless, combining the findings of preattentive bias for threat with the finding of elevated autonomic responses to masked pictorial presentation of threat, one speculation is that the similar response pattern observed on two different outcome measures (cognitive and physiological) in high traitanxious individuals points to the possibility of the involvement of one common underlying factor. Clearly, for this to be corroborated, a study would have to be carried out that compared latency data with autonomic data. One common feature with respect to the two outcome measures is, however, that quantitative differences (threat vs. non-threat) seen in the subliminal exposure condition are more likely to exist in people with elevated anxiety level. Nevertheless, as argued here, these differences are not very likely to occur as a consequence of these individuals evaluating threatening stimuli as more threatening than those at lower levels of anxiety, but rather in the subsequent stage of information-processing, after the valence evaluation phase has occurred. An alternative interpretation would be that some individuals experience difficulty in disengaging from threatening information and, as a consequence, negatively valenced material is not inhibited. Following Bower's (1981) network model, a rapid spread of activation through the entire network will occur if threatening material is not efficiently inhibited. Indeed, anxious people's networks are further assumed to have become larger and more frequently used and, therefore, merely a few matches may be sufficient to cause a massive spread of activation in the memory structure.

Physiological reactivity in response to emotional information has been proposed to serve as an index of activation in the fear/anxiety network (e.g., Lang et al., 1998). As seen in Study II, the increment in SCRs following exposure of masked threatening pictures (compared to neutral) was observed mainly among "true" high trait-anxious individuals. If the reasoning above is correct, for these individuals, unconscious processing of these pictures was sufficient to cause a considerable amount of spread 
of activation in the network, which could be taken as evidence that their fear/anxiety networks were thoroughly assessed. At the same time, these highly threatening pictures are not sufficient to cause a marked increase in SCRs. Low trait-anxious individuals, for example, do indeed also process negatively valenced emotional information unavailable for conscious awareness - in general, this group selectively attend away from masked threat words in the Stroop task. Accordingly, negatively valenced emotional information unavailable for conscious awareness is certainly being processed, but since highly threatening pictures do not produce any marked increase in SCRs, this could, in turn, be taken as evidence that the spread of activation is at best very limited.

\section{Subliminal vs. supraliminal: How much attention should we pay to effects seen in the supraliminal exposure condition?}

In sharp contrast to the subliminal exposure condition, in a consideration of the effects of trait anxiety and social desirability in the supraliminal exposure condition across the studies, there were no consistent response patterns whatsoever. In fact there were only main effects (on threat indices) of these variables in Study II on the autonomic outcome measure, and on only one threat word category on the latency outcome measure in Study IV. One plausible explanation for this is that conscious strategic processes may serve to override the influence of emotionally threatening words and may cancel out any interference effect when the participants are aware of the stimulus content (Williams et al., 1996). In particular, as noted earlier, the Stroop effects in an unmasked, supraliminal exposure condition may differ as a function of conscious strategic processes that are highly variable from one context to another, depending on, for example, pre-experimentally provided information (or other subtle cues). As an illustration, during debriefing several participants spontaneously reported that they attempted to speed up the colour naming whenever a threat word appeared on the screen merely because "they were unpleasant to look at". If such strategies are used, scores obtained in the supraliminal condition should be a less accurate indicator of biased attentional processing. Of course, the absence of other relevant trait and mood measures is a self-evident competing explanation. Even though there is good reason to be preoccupied with a discussion concerning the two tasks measuring selective attention and the effects of trait anxiety on unmasked stimuli, another interesting strategy is to see whether unconscious and conscious perception of threatening material lead to qualitatively different emotional consequences. When response patterns from a supraliminal condition are contrasted with those from a subliminal condition, we are actually examining two different processes, as supported by the fact that interference indices across conditions are at best merely weakly associated. As is evident from Study IV, as well as some previous studies (e.g., MacLeod \& Hagan, 1992; Verhaak et al., 2004), effects from the subliminal exposure condition differ fundamentally from those seen in the supraliminal condition with respect to emotional responses to stressors. Thus far, there is no point in proving an account that selective attention to consciously and 
unconsciously perceived threat originate from the same underlying mechanisms as long as selective attention to unmasked threat cues remain non-predictive of the emotional responses to stressful events. Clearly, there is a need for researchers from several fields to contribute to the literature on cognition and emotion in order to bridge the gap between conscious and unconscious processes, and to more fully understand features that are unique to these different processes.

\section{Limitations of the findings and future directions}

Above, I have provided the general impression of the results based on findings with respect to the high-defensive individuals, as well as some speculations with reference to context (i.e., threat expectations vs. non-threat expectations). However, the generality of these results and speculations is restricted by the measures of social desirability used. Paulhus (1984) demonstrated that social desirability comprises two relatively independent components: self-deceptive enhancement and impression management (i.e., other-deception). Thus, the critical issue here is whether the joint effect of impression management and self-deception (i.e., social desirability) was responsible for the effects seen in the studies in this thesis, or whether these effects depend exclusively on one of these two components of social desirability. Thus, future research should carry out studies comparing the effects of these two components of social desirability on both latency and autonomic data.

The masked Stroop version (i.e., preattentive bias) continues to prove predictive of emotional distress in response to stressful events, but is seemingly predictive of physiological responses to the same stressor as well. Some of the topics in the Introduction were concerned with possible differences between the two different measures of selective attention (i.e., the emotional Stroop and the attentional probe), but also with the threat value of the stimulus material. Accordingly, first, it would be interesting to see whether (or to what extent) the masked version of the attentional probe task predicts emotional responses to stressful events. Second, another interesting aspect is whether and how stronger threat cues will affect the emotional responses compared to mild threat cues. Considering that the Stroop interference effect for words equal the magnitude of effects for pictures (i.e., assuming to possess high threat value), one should perhaps not expect any major change in effects (Kindt \& Brosschot, 1997). However, as this has only been observed with stimuli available for conscious awareness, such an investigation would still be meaningful. Then again, as noted earlier, we do not yet know if the effects of stimulus intensity of the threat cues also hold up in a condition in which these cues cannot be consciously represented. Finally, the effects of preattentive bias on physiological responses to the stressor were obtained on a measure of skin conductance activity. Some studies (e.g., Friedman \& Thayer, 1998) that have investigated the physiological reactivity in response to stressful events in clinical samples have used heart rate variability as a measure of autonomic flexibility. In fact, Turpin (1991) noted that different physiological measures are not usually highly correlated, and that "it should be 
emphasized that no single measure has yet been identified as a psychophysiological index of choice for anxiety" (pp. 367). Thus, future prediction studies would benefit from incorporating a variety of physiological outcome measures in the design.

Although research data could be viewed as holding for both genders, it is important to bear in mind that gender differences in research of cognitive biases have thus far been virtually neglected. In Study I, there were some gender-specific effects with respect to general cognitive performance, but not with respect to content-specific effects. In Study II, exploratory analyses (data not shown) with an eye specifically to gender-specific effects did not indicate that gender matters to any extent. Study III was an all-female sample, and Study IV consisted of only six male participants, which, of course, precluded analyses based on gender. Gender-related effects on the emotional Stroop task have previously been reported in at least two studies (Stewart, et al., 1998; Wikström et al., 2003). With respect to effects of the emotional Stroop task on emotional responses to a stressor, Nay and co-workers (2004) reported stronger associations between the Stroop interference indices and emotional responses for women than was the case for men. Notable in the present context is that there is a predominance of women among those individuals suffering from anxiety disorders (e.g., Barlow, 2002). If preattentive bias is indeed a vulnerability factor in the development of clinical anxiety, could this factor account in part for the preponderance of women among those with anxiety disorders? More focus on possible gender differences is warranted.

There are also certain methodological issues that must be addressed in the future in order to accomplish more consistent effects when using the emotional Stroop task. Because emphasis has been on subliminal presentation in this thesis, do variations in the presentation order for word type (threat and neutral words intermixed, a block of threat words following a block of neutral, or the reversed) affect the outcome, or does the presentation order of the exposure conditions (subliminal following supraliminal trial, or the reversed) exerts influence on each other? There is indeed evidence indicating that subliminal Stroop effects are seen only when subliminal presentation is preceded by supraliminal presentation of the threat words and are, hence, dependent on prior conscious activation of threat-related material (Fox, 1996). Furthermore, these effects were recently replicated by means of another measure of selective attention, namely, the attentional probe task (Luecken, Tartaro, \& Appelhans, 2004). In the latter case, however, an interesting subsidiary finding was that the preceding block of subliminally presented words eliminated the effects seen in the supraliminal exposure condition that were previously seen when no preceding presentation existed. On the other hand, in a study that explicitly tested the possibility that subliminal effects are dependent on pre-activation (Wikström et al., 2003), there was evidence that the effects on the subliminal Stroop can be observed in the absence of a preceding supraliminal presentation of the threat words. Nonetheless, considering the number of studies (e.g., Nay et al., 2004; Pury, 2002; Verhaak et al., 2004) that have employed blocked presentation (participants being randomised to subliminal following supraliminal exposure, or the reverse) which, in 
addition, found effects in accordance with predictions, it seems safe to conclude that the emotional Stroop can reliably capture effects of environmental threat cues occurring outside conscious awareness.

On a more theoretical note, it may prove useful for future studies to examine the possible interactive effect of trait anxiety and anxious mood with respect to effects of threat stimuli that differ in threat value. As I have referred to several times, the Mogg and Bradley (1998) theory states that higher threat value of the stimulus material is associated with increased bias for threat. Could it be the case, for example, that the interactive effect of trait anxiety and anxious mood (as proposed by Williams et al., 1997) will be even more pronounced when highly threatening stimuli are used than is the case for mildly threatening ones (i.e., stronger negative association between threat index and anxious mood for the low trait-anxious, and the reversed for the high trait-anxious)? In addition, because evidence supportive of the view that higher threat value of the stimulus material is associated with increased bias for threat has been achieved only using supraliminal presentation of threat cues, it would be interesting to see if this also holds up in a condition in which the stimulus material cannot be consciously represented.

\section{Concluding remarks}

With an eye specifically to results indicating that a single individual's bias score is not likely to be reproduced from one time to another as well as the influence of worried mood (Study III), but also the somewhat unpredictable outcome for individuals high in defensiveness, there is good reason to agree with Mathews and MacLeod's (2002) claim that "processing biases are anything but fixed" (p. 352), simply because temporary states of mood and contextual cues are likely to influence how the information is processed. As was found in Study IV, but also previously (e.g., Nay et al., 2004), unconscious processing bias of negatively valenced information is associated with emotional distress, and processing bias is causally related to emotional distress (Mathews \& MacLeod, 2002). The fact that the increase in emotional distress could be attributed to processing bias and not pre-existing anxiety levels supports the notion that processing biases might indeed be a key feature in the experience of anxiety as such. That is to say, there might exist a number reasons why biased attentional processing is present: pre-existing enduring anxiety, temporary states of mood, contextual cues, or the interactions between them, and so on. Only processing bias for negatively valenced information, however, seems to be largely responsible for the increment in emotional discomfort during a stressful event.

Considering some complexities involved in this area of research, more information is needed before it can be established that the emotional Stroop task should be viewed as a reliable diagnostic tool for an individual's anxiety status and changes in biased processing, and as a tool in the assessment of vulnerability to clinical anxiety. I have 
tentatively concluded that the outcome is sometimes dependent on temporary states of mood, but some variations in our research environment may also be important. However, the world we live in is also highly variable. We do not, after all, live in laboratory contexts. By being able to pinpoint the factors that are responsible for certain effects on measures of preferential preattentive processing, we are at the same time more likely to translate these factors into something that corresponds to situations in our natural environment with greater confidence. 


\section{References}

Aiken, L. S., \& West, S. G. (1991). Multiple regression: Testing and interpreting interactions. Newsbury Park, CA: Sage.

Amir, N., Elias, J., Klumpp, H., \& Przeworski, A. (2003). Attentional bias to threat in social phobia: Facilitated processing of threat or difficulty disengaging attention from threat? Behaviour Research and Therapy, 41, 1325-1335.

Bargh, J. A., \& Ferguson, M. J. (2000). Beyond behaviorism: On the automaticity of higher mental processes. Psychological Bulletin, 126, 925-945.

Barlow, D. H. (2002). Anxiety and its disorders (2nd ed.). New York: Guilford Press

Baumeister, R. F., \& Cairns, K. J. (1992). Repression and self-presentation: When audiences interfere with self-deceptive strategies. Journal of Personality and Social Psychology, 62, 51-862.

Beck, A. T., \& Clark, D. M. (1997). An information processing model of anxiety: Automatic and strategic processes. Behaviour Research and Therapy, 35, 49-58.

Borkovec, T. D. (1994) The nature, functions, and origins of worry. In: G. C. L. Davey and F. Tallis, Editors, Worrying: perspectives on theory, assessment and treatment (pp. 5-33). John Wiley \& Sons Ltd.

Bower, G. H. (1981). Mood and memory. American Psychologist, 36, 129-148.

Bradley, B. P., Mogg, K., Falla, S. J., \& Hamilton, L. R. (1998). Attentional bias for threatening facial expressions in anxiety: Manipulation of stimulus duration. Cognition and Emotion, 12, 737-753.

Bradley, B. P., Mogg, K., White, J., \& Millar, N. (1995). Selective processing of negative information: Effects of clinical anxiety, concurrent depression, and awareness. Journal of Abnormal Psychology, 104, 532-536.

Brosschot, J, F., de Ruiter, C., \& Kindt, M. (1999). Processing bias in anxious subjects and repressors, measured by emotional Stroop interference and attentional allocation. Personality and Individual Differences. 26, 777-793.

Calvo, M. G., \& Eysenck, M. W. (2000). Early vigilance and late acoidance of threat processing: Repressive coping versus low/high anxiety. Cognition and Emotion, 14, 763-787.

Cattell, R. B., Cattell, A. K., \& Cattell, H. E. (1993). Sixteen Personality Factor Questionnaire, Fifth Edition. Champaign, IL: Institute for Personality and Ability Testing, Inc. 
Chen, E., Lewin, M. R., \& Craske, M. G. (1996). Effects of state anxiety on selective processing of threatening information. Cognition and Emotion, 10, 225-240.

Cheesman, J., \& Merikle, P. M. (1986). Distinguishing conscious from unconscious perceptual processes. Canadian Journal of Psychology, 40, 343-367.

Clark, L. A., Watson, D., \& Mineka, S. (1994). Temperament, personality, and the mood and the anxiety disorders. Journal of Abnormal Psychology, 103, 103-116.

Cohen, J., \& Cohen, P. (1983). Applied multiple regression/correlation analysis for behavioral sciences (2nd ed.). Hillsdale, NJ: Erlbaum.

Cohen, J. D., Dunbar, K., McClelland, J. L. (1990). On the control of automatic processes: A parallel distributed processing account of the Stroop effects. Psychological Review, 97, 332-361.

Crowne, D. P., \& Marlowe, D. (1964). The approval motive: Studies in evaluative dependence. New York: Wiley.

Dalgleish, T. (1995). Performance on the emotional Stroop task in groups of anxious, experts, and control subjects: A comparison of computer and card presentation formats. Cognition and Emotion, 9, 341-362.

Davis. P.J., \& Schwartz. G.E. (1987). Repression and the inaccessibility of affective memories. Journal of Personality and Social Psychology, 52, 155-162.

Dawkins, K. \& Furnham, A. (1989). The colour naming of emotional words. British Journal of Psychology, 80, 383-389.

Derakshan, N., \& Eysenck, M. W. (1997). Repression and repressors: Theoretical and experimental approaches. European Psychologist, 2, 235-246.

Derakshan, N., \& Eysenck, M. W. (2005). When the bogus pipeline interferes with selfdeceptive strategies: Effects of state anxiety in repressors. Cognition and Emotion, 19, 83-100.

Derryberry, D., \& Reed, M. A. (2002). Anxiety-related attentional biases and their regulation by attentional control. Journal of Abnormal Psychology, 11, 225-236.

Devineni, T., Blanchard, E. B., Hickling, E. J., \& Buckley, T. C. (2004). Effects of psychological treatment on cognitive bias in motor vehicle accident-related posttraumatic stress disorder. Anxiety Disorders, 18, 211-231.

de Ruiter, C., \& Brosschot, J. F. (1994). The emotional Stroop effect in anxiety: Attentional bias or cognitive avoidance? Behaviour Research and Therapy, 32, 315-319. 
Egloff, B., \& Hock, M. (2001). Interactive effects of state anxiety and trait anxiety on emotional Stroop interference. Personality and Individual Differences, 31, 875-882.

Egloff, B., \& Hock, M. (2003). Assessing attention allocation toward threat-related stimuli: A comparison of the emotional Stroop task and the attentional probe task. Personality and Individual Differences, 35, 475-483.

Eysenck, M. W. (1997). Anxiety and cognition: A unified theory. Hove, UK: Psychology press.

Fox, E. (1994). Attentional bias in anxiety: A defective inhibition hypothesis. Cognition and Emotion, 8, 165-195.

Fox, E. (1996) Selective processing of threatening words in anxiety: The role of awareness. Cognition and Emotion, 10, 449-480.

Fox, E., Russo, R., Bowles, R., \& Dutton. (2001). Do threatening stimuli draw or hold visual attention in subclinical anxiety? Journal of Experimental Psychology, 130, 681-700.

Fox, E., Russo, R., \& Dutton. (2002). Attentional bias for threat: Evidence for delayed disengagement from emotional faces. Cognition and Emotion, 16, 355-379.

Friedman, B. H., \& Thayer, J. F. (1998). Anxiety and autonomic flexibility: A cardiovascular approach. Biological Psychology, 49, 303-323.

Greenwald, A. G. (1992). New look 3: Unconscious cognition reclaimed. American Psychologist, 47, 766-779.

Hafer, C. L. (2000). Do innocent victims threaten the belief in a just world? Evidence from a modified Stroop task. Journal of Personality and Social Psychology,79, 165-173.

Hoehn-Saric, R., \& McLeod, D. R (2000). Anxiety and arousal: Physiological changes and their perception. Journal of Affective Disorders, 61, 217-224.

Jezova, D., Makatsori, A., Duncko, R., Moncek, F., \& Jakubek, M. (2004). High trait anxiety in healthy subjects is associated with low neuroendocrine activity during psychosocial stress. Progression Neuro-Psychopharmacology \& Biological Psychiatry, 28, 1331-1336.

Kindt, M., Bierman, D., \& Brosschot, J. F. (1996). Stroop versus Stroop: Comparison of the card format and a single-trial format of the standard color-word Stroop task and the emotional Stroop task. Personality and Individual Differences, 21, 653-661.

Kindt, M., Bierman, D., \& Brosschot, J. F. (1997). Cognitive bias in spider fear and control children: Assessment of emotional interference by card format and a single trial format of the Stroop task. Journal of Experimental Child Psychology, 66, 163-179. 
Kindt, M., \& Brosschot, J. F. (1997). Phobia-related cognitive bias for pictorial and linguistic stimuli. Journal of Abnormal Psychology, 106, 644-648.

Kindt, M., \& Van den Hout, M. (2001). Selective attention and anxiety: A perspective on developmental issues and the casual status. Journal of Psychopathology and Behavioural Assessment, 23, 193-202.

Kline, J. P., Blackhart, G. C., \& Joiner, T. E. (2002). Sex, lie scales, and electrode caps: An interpersonal context for defensiveness and anterior electroencephalographic asymmetry. Personality and Individual Differences, 33, 459-478.

Kline, J. P., Knapp-Kline, K., Schwartz, G. E. R., \& Russek, L. G. S. (2001). Anterior symmetry, defensiveness, and perception of parental caring. Personality and Individual Differences, 31, 1135-1145.

Koster, E. H. W., Verschuere, B., Crombez, G., \& van Damme, S. (2004). Time-course of attention for threatening pictures in high and low trait anxiety. Behaviour Research and Therapy, 43, 1087-1098.

Lane, R. D., Merikangas, K. R., Schwartz, G. E., Huang, S. S., \& Prusoff, B. A. (1990). Inverse relationship between defensiveness and lifetime prevalence of psychiatric disorder. American Journal of Psychiatry, 147, 573-578.

Lang, P. J., Bradley, M. M., \& Cuthbert, B. N. (1998). Emotion, motivation, and anxiety: Brain mechanisms and psychophysiology. Biological Psychiatry, 44, 1248-1263.

Lang, P. J., Bradley, M. M., \& Cuthbert, B. N. (1999). International affective picture system (IAPS): Instruction manual and affective ratings. Technical report A-4, The Center for Research in Psychophysiology; University of Florida.

Lazarus, R. S. (1999). The cognition-emotion debate: A bit of history. In: T. Dalgleish and M. Power, Editors, Handbook of cognition and emotion, Wiley, New York.

LeDoux, J. E. (1994). Emotion, memory and the brain. Scientific American, 270, 32-39.

Lewicki, P., Hill, T., \& Czyzewska, M. (1992). Nonconscious acquisition of information. American Psychologist, 47, 796-801.

Loftus, E. F., \& Klinger, M. R. (1992). Is the unconscious smart or dumb? American Psychologist, 47, 761-765.

Luecken, L. J., Tartaro, J., \& Appelhans, B. (2004). Strategic coping responses and attentional biases. Cognitive Therapy and Research, 28, 23-37. 
Lundh, L .G., Wikström, J., \& Westerlund, J. (2001). Cognitive bias, emotion and somatic complaints in a normal sample. Cognition and Emotion, 15, 249-277.

Lundh, L. G., Wikström, J., Westerlund, J., \& Öst, L. G. (1999). Preattentive bias for emotional information in panic disorder with agoraphobia. Journal of Abnormal Psychology, 108, 222-232.

Mackintosh, B., \& Mathews, A. (2003). Don't look now: Attentional avoidance of emotionally-valenced cues. Cognition and Emotion, 17, 623-646.

MacLeod, C., \& Hagan, R. (1992). Individual differences in the selective processing of threatening information, and the emotional responses to a stressful life event. Behaviour Research and Therapy, 30, 151-161.

MacLeod, C., \& Mathews, A. (1988). Anxiety and the allocation of attention to threat. Quarterly Journal of Experimental Psychology: Human Experimental Psychology; 40, 653670.

MacLeod, C., \& Rutherford, E. M. (1992). Anxiety and the selective processing of emotional information: Mediating roles of awareness, trait and state variables, and personal relevance of stimulus materials. Behaviour Research and Therapy, 30, 479-491.

MacLeod, C., Rutherford, E., Campbell, L., Ebsworthy, G., \& Holker, L. (2002). Selective attention and emotional vulnerability: Assessing the causal basis of their association through the experimental manipulation of attentional bias. Journal of Abnormal Psychology, 11, 107-123.

McNally, R. (1989). Is anxiety sensitivity distinguishable from trait anxiety? Reply to Lillenfield, Jacob, and Turner (1989). Journal of Abnormal Psychology, 98, 193-194.

McNally, R. J. (1990). Psychological approaches to panic disorder: A review. Psychological Review, 108, 403-419.

Mansell, W., Clark, D. M., Ehlers, A., \& Chen, Y. P. (1999). Social anxiety and attention away from emotional faces. Cognition and Emotion, 13, 673-690.

Mathews, A. (1990). Why worry? The cognitive function of anxiety. Behaviour Research and Therapy, 28, 455-468.

Mathews, A. (2004). On the malleability of emotional encoding. Behaviour Research and Therapy, 42,1019-1036.

Mathews, A., \& Mackintosh, B. (1998). A cognitive model of selective processing in anxiety. Cognitive Therapy and Research, 22, 539-560. 
Mathews, A., \& MacLeod, C. (2002). Induced processing biases have causal effects on anxiety. Cognition and Emotion, 16, 331-354.

Mattia, J. I., Heimberg, R. G., \& Hope, D. A. (1993). The revised Stroop color-naming task in social phobics. Behaviour Research and Therapy, 31, 305-313.

Merikle, P. M., \& Joordens, S. (1997). Parallels between perception without attention and perception without awareness. Consciousness and Cognition, 6, 219-236.

Merikle, P. M., \& Reingold, E. M. (1992). Measuring unconscious perceptual processes. In R. F. Bornstein \& T. S. Pittman (Eds.), Perception without awareness: Cognitive, clinical, and social perspectives (pp. 55-80). New York: Guilford.

Mogg, K., \& Bradley, B. P. (1998). A cognitive-motivational analysis of anxiety. Behaviour Research and Therapy 36, 809-848.

Mogg, K., \& Bradley, B. P. (1999). Orienting of attention to threatening facial expressions presented under conditions of restricted awareness. Cognition and Emotion, 13, 713740 .

Mogg, K., \& Bradley, B. P. (2002). Selective orienting of attention to masked threat faces in social anxiety. Behaviour Research and Therapy 40, 1403-1414.

Mogg, K., Bradley, B. P., Dixon, C., Fisher, S., Twelftree, H., \& McWilliams, A. (2000a). Trait anxiety, defensiveness and selective processing of threat: An investigation using two measures of attentional bias. Personality and Individual Differences, 28, 10631077.

Mogg, K., Bradley, B. P., Miles, F., \& Dixon, R. (2004). Time course of attentional bias for threat scenes: Testing the vigilance-avoidance hypothesis. Cognition and Emotion, 18, 689-700.

Mogg, K., Bradley, B. P., Millar, N., \& White, J. (1995). A follow-up study of cognitive bias in generalized anxiety disorder. Behaviour Research and Therapy, 33, 927-935.

Mogg, K., Bradley, B. P., Williams, R., \& Mathews, A. (1993). Subliminal processing of emotional information in anxiety and depression. Journal of Abnormal Psychology, 102, 304-310.

Mogg, K., McNamara, J., Powys, M., Rawlinson, H., Seiffer, A., \& Bradley, B. P. (2000b). Selective attention to threat: A test of two cognitive models of anxiety. Cognition and Emotion, 14, 375-399.

Mogg, K., Philippot, P., \& Bradley, B. P. (2004). Selective attention to angry faces in clinical social phobia. Journal of Abnormal Psychology, 113, 160-165. 
Morris, J. S., Öhman, A., \& Dolan, R. J. (1998). Conscious and unconscious emotional learning in the amygdala. Nature, 393, 467-470.

Musa, C., Lépine, J. P., Clark, D. M., Mansell, W., \& Ehlers, A. (2003). Selective attention in social phobia and the moderating effect of a concurrent depressive disorder. Behaviour Research and Therapy, 41, 1043-1054.

Myers, L. B., \& McKenna, F. P. (1996). The colour naming of socially threatening words. Personality and Individual Differences, 20, 801-803.

Najström, M., \& Högman, L. (2003). Skin conductance response in Swedish United Nations soldiers exposed to fear-relevant stimuli. Cognitive Behaviour Therapy, 32, 161165.

Nay, W. T., Thorpe, G. L., Roberson-Nay, R., Hecker, J. E., \& Sigmon, S. T. (2004). Attentional bias to threat and emotional response to biological challenge. Anxiety Disorders, 18, 609-627.

McNaughton, N., \& Gray, J. A. (2000). Anxiolyctic action on behavioural inhibition system implies multiple types of arousal contribute to anxiety. Journal of Affective Disorders, 61, 161-176.

Newman, L. S. \& McKinney, L. C. (2002). Repressive coping and threat avoidance: An idiographic Stroop study. Personality and Social Psychology Bulletin, 28, 409-422.

Öhman, A. (1996). Preferential preattentive processing of threat in anxiety: Preparedness and attentional biases. In R. M. Rapee (Ed.), Current controversies in the anxiety disorders (pp 253 - 290). The Guilford Press.

Öhman, A. (1997). Unconscious pre-attentive mechanisms in the activation of phobic fear. In G. C. L. Davey (Ed.), Phobias-a handbook of theory, research and treatment (pp. 349374). John Wiley \& Sons Ltd.

Öhman, A., \& Mineka, S. (2001). Fears, phobias, and preparedness: Toward an evolved module of fear and fear learning. Psychological Review, 108, 483-522.

Öhman, A., \& Soares, J. J. F. (1993). On the automatic nature of phobic fear: Conditioned electrodermal responses to masked fear-relevant stimuli. Journal of Abnormal Psychology, 103, 1221-1232.

Öhman, A., \& Soares, J. J. F. (1994). "Unconscious Anxiety": Phobic responses to masked stimuli. Journal of Abnormal Psychology, 103, 231-240.

Oldenburg, C., Lundh, L. G., \& Kivistö, P. (2002). Explicit and implicit memory bias, trait anxiety, and repressive coping style. Personality and Individual Differences, 32, 107-119. 
Paulhus, D. L. (1984). Two components models of socially desirable responding. Journal of Personality and Social psychology, 46, 598-609.

Pury, C. S. (2002). Information processing predictors of emotional response to stress. Cognition and Emotion, 16, 667-683.

Putman, P., Herman, E., \& van Honk, J. (2004). Emotional Stroop performance for masked angry faces: It's BAS, not BIS. Emotion, 4, 305-311.

Rapee, R. M., \& Heimberg, R. G. (1997). A cognitive-behavioral model of anxiety in social phobia. Behaviour Research and Therapy, 35, 741-756.

Russell, M .T., \& Karol, D. L. (1997). 16PF: Manual: $5^{\text {th }}$ version. Stockholm: Psykologiförlaget $\mathrm{AB}$.

Rutherford, E. M., MacLeod, C., \& Campbell, L. (2004). Negative selectivity effects and emotional selectivity effects in anxiety: Differential attentional correlates of state and trait variables. Cognition and Emotion, 18. 711-720.

Schalling, D. (1985). Personality correlates of elevated blood pressure. Anxiety, unexpressed anger, and lack of assertiveness. In C. D. Spielberger, I. G. Sarason, \& P. B. Defares (Eds.), Stress and anxiety. Volume 9. Washington: Hemisphere, pp. 241-251.

Schwartz, G. E. (1990). Psychobiology of repression and health: A systems approach. In J. L. Singer (Ed.), Repression and Dissociation (pp. 405-434). Chicago: University of Chicago press.

Silverman, L. H., Lachman, F. M., \& Milich, R. H. (1982). The search for oneness. New York: International Univ Press.

Silvert, L., Delplanque, S., Bouwalerh, H., Verpoort, C., \& Sequire, H. (2004). Autonomic responding to aversive words without conscious valence discrimination. International Journal of psychophysiology, 53, 135-145.

Sohlberg, S., \& Birgegard, A. (2003). Persistent complex subliminal activation effects: First experimental observations. Journal of Personality and Social Psychology, 85, 302-316.

Spielberger, C. D., Gorsuch, R. C., Lushene, R. E., Vagg, P. R., \& Jacobs, G. A. (1983). Manual for the State-Trait Anxiety Inventory. Palo Alto, CA: Consulting Psychologists Press.

StatSoft (1994). Statistica for the Macintosh. Tulsa, OK: Author.

Stewart, S. H., Conrod, P. J., Gignac, M. L., Phil, R. O. (1998). Selective processing biases in anxiety-sensitive men and women. Cognition and Emotion, 12, 105-133. 
Sutton, S. K., \& Davidson, R. J. (2000). Prefrontal brain electrical asymmetry predicts the evaluation of affective stimuli. Neuropsychologia 38, 1723-1733

Thayer, J. F., \& Friedman, B. H. (2002). Stop that! Inhibition, sensitization, and their neurovisceral concomitants. Scandinavian Journal of Psychology, 43, 123-130.

Thayer, J. F., Friedman, B. H., \& Borkovec, T. D. (1996). Autonomic characteristics of generalized anxiety disorder and worry. Biological Psychiatry, 39, 255-266.

Tomarken. A. J., \& Davidson, R. J. (1994). Frontal brain activation in repressors and nonrepressors. Journal of Abnormal Psychology, 103, 339-349.

Turpin, G. (1991). The psychophysiological assessment of anxiety disorders: Three-systems measurement and beyond. Psychological Assessment: A Journal of Consulting and Clinical Psychhology, 3, 366-375.

van Honk, J., Tuiten, A., Van den Hout, M., Putman, P., de Haan, E., \& Stam, H. (2001). Selective attention to unmasked and masked threatening words: Relationships to trait anger and anxiety. Personality and Individual Differences, 30, 711-720.

Van den Hout, M., Tenney, N., Huygens, K., \& de Jong, P. (1997). Preconscious processing bias in specific phobia. Behaviour Research and Therapy 35, 29-34.

Van den Hout, M., de Jong, P., \& Kindt, M. (2000). Masked fear words produce increased SCRs: An anomaly for Öhman's theory of pre-attentive processing in anxiety. Psychophysiology, 37, 283-288.

Van den Hout, M., Tenney, N., Huygens, K., Merckelbach, H., \& Kindt, M. (1995). Responding to subliminal threat cues is related to trait anxiety and emotional vulnerability: A successful replication of MacLeod and Hagan (1992). Behaviour Research and Therapy, 33, 451-454.

Verhaak, C. M., Smeenk, J. M. J., van Minnen, A., \& Kraaimat, F. W. (2004). Neuroticism, preattentive and attentional biases towards threat, and anxiety before and after a severe stressor: A prospective study. Personality and Individual Differences, 36, 767-778.

Weinberger, D. A. (1990). The construct validity of the repressive coping style. In J. L. Singer (Ed.), Repression and Dissociation (pp. 337-386). Chicago: University of Chicago press.

Weinberger, D. A., Schwartz, G. E., \& Davidson, J. R. (1979). Low-anxious, high-anxious, and repressive coping styles: psychometric patterns and behavioural and physiological responses to stress. Journal of Abnormal Psychology, 88, 369-380. 
Wikström, J., Lundh, L. G., \& Westerlund, J. (2003). Stroop effects for masked threat words: Preattentive bias or selective awareness? Cognition and Emotion, 17, 827-842.

Wilken, J. A., Smith, B. D., Tola, K., \& Mann, M. (2000). Trait anxiety and prior exposure to non-stressful stimuli: Effects on psychophysiological arousal and anxiety. International Journal of Psychophysiology, 37, 233-242.

Williams, J. M. G., Watts, F. N., MacLeod, C., \& Mathews, A. (1988). Cognitive psychology and emotional disorders. Chichester: Wiley.

Williams, J. M. G., Mathews, A., \& MacLeod, C. (1996). The emotional Stroop task and psychopathology, Psychological Bulletin, 120, 3-24.

Williams, J. M. G., Watts, F. N., MacLeod, C., \& Mathews, A. (1997). Cognitive psychology and emotional disorders, 2nd ed. New York: Wiley.

Wilson, E., \& MacLeod, C. (2003). Contrasting two accounts of anxiety-linked attentional bias: Selective attention to varying levels of stimulus threat intensity. Journal of Abnormal Psychology, 112, 212-218.

Yiend, J., \& Mathews, A. (2001). Anxiety and attention to threatening pictures. The Quarterly Journal of Experimental psychology, 54, 665-681. 